\title{
Numerical Simulation of the Impact of Geological Heterogeneity on Performance and Safety of THAI Heavy Oil Production Process
}

\author{
Muhammad Rabiu Ado ${ }^{\mathrm{a}, \#}$, Malcolm Greaves ${ }^{\mathrm{b}}$, and Sean P. Rigby, \\ ${ }^{a}$ Department of Chemical and Environmental Engineering, University of Nottingham, \\ University Park, Nottingham, NG7 2RD, U.K. \\ ${ }^{b}$ Department of Chemical Engineering, University of Bath, Claverton Down, Bath, BA2 7AY \\ U.K. \\ * Corresponding author:
}

Tel: +44 (0) 115951 4081, Email: enzspr@exmail.nottingham.ac.uk

\# Current address:

Department of Chemical Engineering, College of Engineering, King Faisal University, P.O. Box: 380, Al-Ahsa 31982, Kingdom of Saudi Arabia.

\begin{abstract}
The Toe-to-Heel Air Injection (THAI) in-situ combustion process is an efficient way to extract heavy oil and bitumen. However, such reservoirs are often geologically heterogeneous. This work studied the impact of a range of different geological heterogeneities, often found in bitumen deposits, on the performance and safety of THAI. These heterogeneities included random heterogeneity, layered reservoirs, shaly reservoirs, and semi-permeable cap-rocks. A further aim was to also develop potential remedial measures, such as selective well placement. It was found that the degree of symmetry assumed for the reservoir model had a substantial impact on the predicted level of oil production. This is of particular relevance to otherwise apparently symmetrical well placement designs such as staggered line drive. While the presence of impermeable zones resulted in the decrease in the overall oxygen utilisation for shaly reservoirs, compared to simply low permeability reservoirs, there was no evidence of oxygen breakthrough due to preferential channelling into the production well. In layered reservoirs, the development of a rich oil bank during THAI operation depended upon the distribution of permeability around the horizontal producer (HP), and did not occur when there was high permeability just above the HP. It has been shown that the proper representation of the cap-rock in reservoir models for the simulation of THAI is essential in order to accurately mimic the full pattern of heat distribution into the oil zone of the reservoir, and, thence, fuel lay-down. While THAI can operate stably with a permeable cap-rock, vertical permeabilities above $\sim 1-3 \mathrm{mD}$ led to significant loss of combustion gases from the reservoir.
\end{abstract}

Keywords: bitumen; shale inter-layer; heterogeneity; cap-rock; thief-zone; THAI 


\section{INTRODUCTION}

In order to enable the transition to more sustainable fuels, new fossil fuel resources will be required to meet growing energy demands in the meantime. Heavy oil reserves are approximately twice those of lighter oils (Ravalec et al., 2009). However, the high viscosity of such oils makes them very difficult to recover. Thermal methods have provided an efficient way to extract heavy oil and bitumen. However, the performance of such methods depends upon the nature of the geology of the hydrocarbon deposits. This is because many thermal methods rely upon gravity drainage, so that any geological heterogeneities will likely have a significant effect on flow directionality. There have been many previous studies that have looked at the impact of geological heterogeneity in oil sands reservoirs on the performance of some thermal extraction methods, like SAGD and CSS (Ravalec et al., 2009; Su et al., 2013; Nguyen et al., 2014), but there has been very little investigation of the THAI process, in this respect. Typically, past studies of the THAI process assumed regular, homogeneous oil sands beds.

A key feature of oil sand deposits, such as in the Lower Cretaceous Middle McMurray Formation in north-eastern Alberta, is the presence of shale inter-layers ( $\mathrm{Su}$ et al., 2013). The impact of these geological features on steam-assisted gravity drainage (SAGD) and VAPEX has been studied by experiment and simulations. From simulation studies, Ravalec et al. (2009) showed that shale layers impede the development of the steam chamber in SAGD. However, they found that the well placement was critical, since shale layers could be bypassed to provide a more optimal solution. Fatemi (2012) used simulations to show that the performance of SAGD was lower in the presence of continuous and longer shale layers. In more recent simulations of SAGD using highly detailed and realistic geological models from the McMurray Formation, Su et al. $(2013,2014)$ also showed that the well placement relative to the geology made a significant difference. They found that well-pairs placed perpendicular to shale layers performed better than well-pairs orientated parallel to the shale layers because in the former case the wells cut across the impermeable shale thereby allowing steam to be injected in otherwise poorly accessible regions. 
The impact of other types of geological feature, such as reservoir permeability variation, and the presence of the fracture networks, on the SAGD process has also been studied. Simulations have found that the high conductivity of vertical fractures can help steam to propagate more deeply into the reservoir, and then diffuse out and affect a wider region, and so the formation of the steam chamber in SAGD is faster in fractured beds (Nguyen et al., 2014). However, in contrast, the presence of horizontal fractures was found to lead to reduced cumulative oil production.

Toe-to-heel air injection (THAI) is a variant of conventional in-situ combustion (ISC) that uses a horizontal production well to recover mobilized partially upgraded heavy oil. The Whitesands THAI field pilot at Conklin, Alberta Canada, operated for five years by Petrobank, upgraded the bitumen by up to 4.5 API points (Petrobank, 2009). In addition, the THAI pilot run by Petrobank at the conventional heavy oil reservoir of Kerrobert, Saskatchewan, Canada, initially produced oil upgraded by up to nearly 6-8 API points (Petrobank, 2008). The follow-on semi-commercial expansion, started in 2009, achieved in-situ upgrading in the range 4-6 API points. This represents significant 'added value' to producers of lower value heavy oil and bitumen, and currently heavy oil discounts are as high as $50 \%$. THAI has a number of advantages over other heavy oil recovery techniques, such as high energy efficiency (Rabiu Ado et al., 2017). However, it is necessary to understand how the process can be run efficiently in reservoirs with geological heterogeneity.

Hence, as a first step, therefore, a detailed study will be made of a laboratory-scale THAI process model for virgin Athabasca Oil Sands bitumen for reservoirs with various types of geological heterogeneity. In particular, given the aforementioned findings concerning impact of geological heterogeneity on SAGD, it is necessary to investigate the effects these heterogeneities would have on THAI, for which the mechanism of operation is quite different. Previous work has also 
shown the ability of simulations to test a wider variety of geological features than would be practicable by experiment, and thus that approach will be used here. In order that the results have the broadest applicability possible, the models tested will have generic types of heterogeneity, rather than trying to represent the particular geology of specific fields, such that general trends might be extracted. Simulations also permit the safe testing of potentially hazardous scenarios. A key issue that will be studied is the impact of heterogeneity on oxygen utilisation and break-through. An important issue for safety is the quality of seal provided by the reservoir cap-rock. In addition, the role of geological heterogeneity in scale-up will also be considered.

\section{MODEL DEVELOPMENT}

In previous work (Rabiu Ado et al., 2017), a CMG STARS ${ }^{\circledR}$ simulation model for THAI has been developed and validated against a laboratory-scale experimental study. Since this model has been shown to be capable of closely matching both continuous and transient aspects of the performance of THAI in the laboratory, where phenomena can be observed in much greater detail than in the field, it will be used as the basis to test the influence of most of the different types of geological heterogeneity considered below. Many forms of geological heterogeneity exhibit fractal behaviour, whereby structural characteristics repeat over several length-scales even down to the micro-scale (Barr, 2007). Hence, results for the laboratory-scale will potentially be readily scale-able to larger length-scales.

A detailed description of the model, including oil pyrolysis and combustion kinetics, can be found in earlier work (Rabiu Ado et al., 2017), and thus will not be repeated here. A gridblock refinement study was also conducted in this previous work, and the appropriate gridblock size was also determined. It is also noted that geological heterogeneity within the reservoir itself can only be specified by being made up of blocks of the simulation matrix, and, the larger the dimensions of the matrix, the smaller the volume of each gridblock. Increasing the volume of each gridblock to represent field scale will compromise the accuracy of the result because this limits the detail with which the physicochemical 
processes of the combustion front that can be captured by the simulation. Hence, the geological heterogeneity within the reservoir was simulated with a laboratory scale matrix. However, for the model of the reservoir with a gas overlayer, the heterogeneity does not lie within the oil sands reservoir region matrix and thus does not limit the volume of each gridblock. Hence, the gas overlayer model can be simulated at the field scale using a previously derived model (Rabiu Ado, 2017). The initial oil saturation was $85 \%$.

In this work, the types of heterogeneities tested are models (in the literal scientific sense) in that they capture the essential, primary, elemental features of particular classes of heterogeneity, but leaving out the idiosyncratic second order variations likely in natural heterogeneities.

\subsection{Heterogeneous reservoirs}

A laboratory scale model, which is similar in dimensions to that used in previous work (Rabiu Ado et al., 2017) was used for the study of the effect of reservoir heterogeneity on the THAI process (see Figure 1). In this particular case, this is because representing the heterogeneity on gridblocks of the same length as the thickness of the combustion front will not only capture the physico-chemical processes, but it will also reveal the potential for alteration to the continuity of the combustion front, which was observed in the homogeneous case. However, prior to this, the sensitivity of the three-dimensional combustion cell model to different absolute permeability values was investigated.

In order to simulate the laboratory scale models, the no flux boundary condition was assumed all over the boundary of the models, except via the horizontal injector (HI) and the horizontal producer (HP). This is in accordance with what was reported experimentally (Rabiu Ado et al., 2017). The HI well 
was flow-controlled with the air injection rate at the early stage set to $8000 \mathrm{~cm}^{3} \mathrm{~min}^{-1}$, before it was increased by one-third to $12,000 \mathrm{~cm}^{3} \mathrm{~min}^{-1}$ at $190 \mathrm{~min}$, and then maintained until $320 \mathrm{~min}$. A bottom hole pressure (BHP) of $200 \mathrm{kPa}$ and a total liquid production rate of $25 \mathrm{~cm}^{3} \mathrm{~min}^{-1}$ were respectively specified as the primary and secondary boundary conditions for the HP well. When the maximum BHP was violated, then the flow rate became the primary variable to enforce. For example, when BHP was going to be $>200 \mathrm{kPa}$, the flow rate was then controlled to maintain the BHP as specified. It was assumed that, during the experiment, heat loss only occurred from via both overburden and underburden. Therefore, the heat loss parameters were selected via trial and error until all the experimentally measured performance parameters of the THAI process are matched (Ado et al., 2018).

Figure 1. Combustion cell dimensions and injector and producer wells arrangement for heterogeneous reservoir model

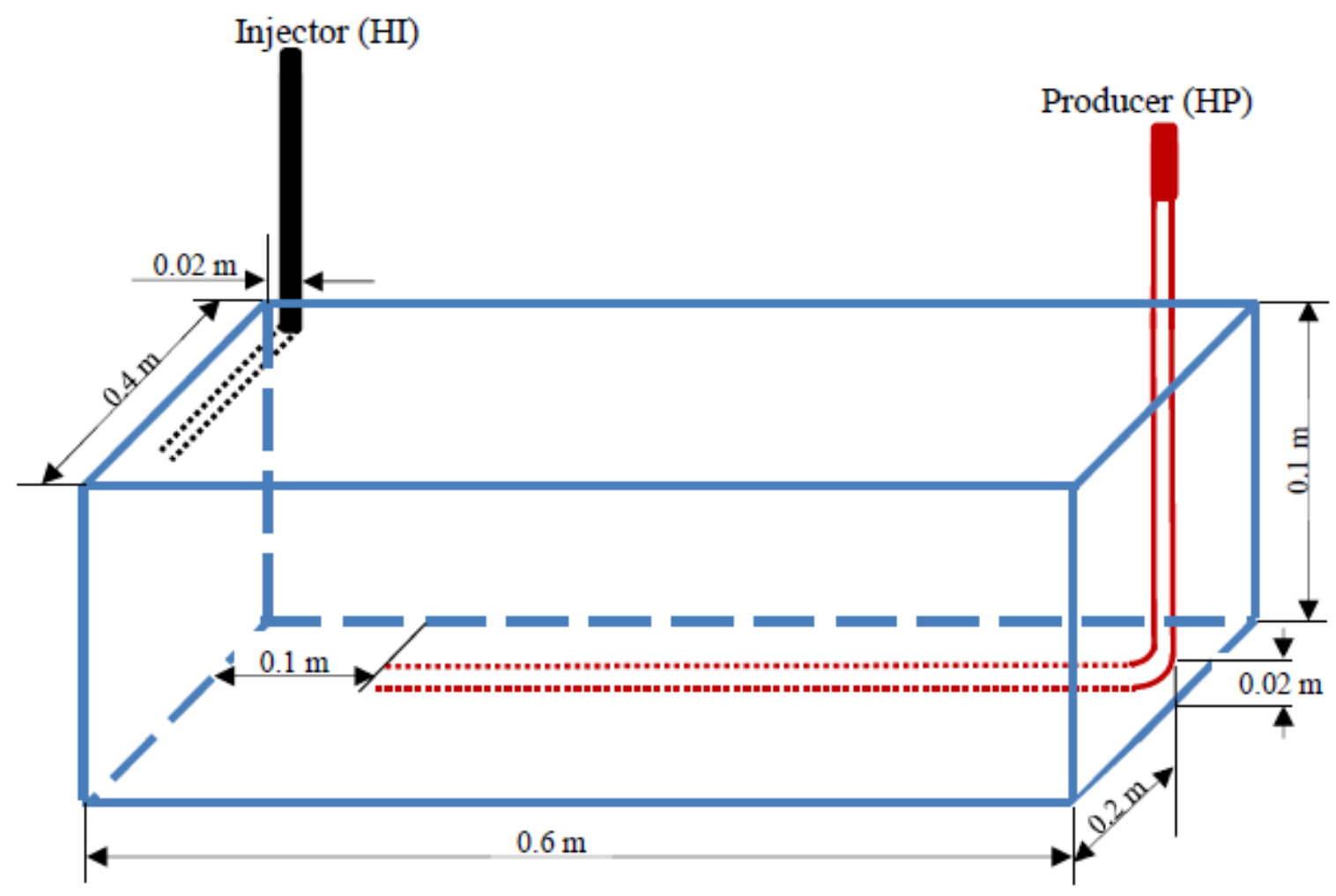




\subsubsection{Base case homogeneous model with constant $K_{v} / K_{h}$}

The initial validation of the model (i.e. the base case) in previous work (Rabiu Ado et al., 2017) was made with horizontal, $K_{h}$, and vertical, $K_{v}$, absolute permeabilities of 11500 and $3450 \mathrm{md}$, respectively (i.e. $\mathrm{K}_{\mathrm{v}} / \mathrm{K}_{\mathrm{h}}=0.3$ ). These values of permeability are characteristic of the sandpack used in laboratoryscale experiments. In the new work presented here, three different runs were performed under the same conditions as the base case but using $K_{h}$ values of 8625,5750 , and $2875 \mathrm{md}$. This allowed the variation of the fractional oil recovery, cumulative air to oil ratio (CAOR), etc. with absolute permeability to be determined.

\subsubsection{Random reservoir heterogeneity with constant $K_{\vee} / K_{h}$}

Since reservoir heterogeneity will increase tortuosity for flow this may impact oil production. In order to test reservoir heterogeneity, the laboratory-scale model was discretised into $58 \times 37 \times 7$ grid blocks, but with further refinement close to the wellbore region, such that the total number of grid blocks in the computational domain became 16300.

The Matlab functionality, rand, was used to generate a uniform distribution of random values, over the interval of 0 to $11500 \mathrm{md}$, which were allocated to the grid blocks. The resulting $259 \times 58$ matrix of the uncorrelated white noise permeability field represents $K_{h}$, while the ratio $K_{\downarrow} / K_{h}=0.3$ was maintained at every point in the model. The random uncorrelated heterogeneity model is shown in Figure 2. The model was run under the same conditions as in the homogeneous case. 
Figure 2. Uncorrelated 'white noise' permeability field model for a randomly heterogeneous reservoir

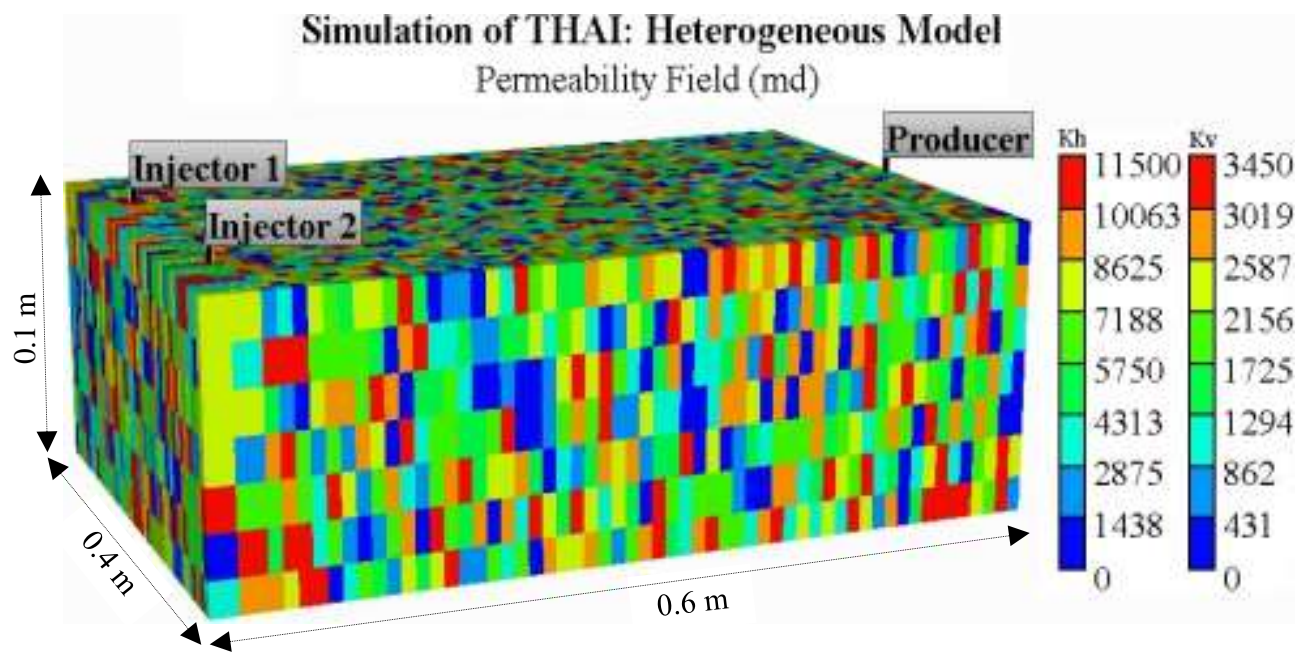

\subsubsection{Dual porosity reservoir}

Using the same permeability realisation as shown in Figure 2, the effect of randomly distributed low permeability zones on THAI was also investigated. However, in this case, the reservoir models were obtained by assuming that a certain proportion of the reservoir volume was occupied by low permeability zones. This is similar to the dual porosity model used by Nguyen et al. (2014) to study performance of SAGD. Figure 3(a) shows the permeability field in which the probability $\left(\mathrm{P}_{30}\right)$ of finding low permeability zones at any given point is taken to be at most $30 \%$. Since a uniform random distribution was used to generate the permeability field, it then follows that any location which has a permeability value of $\leq 30 \%$ of the maximum $K_{h}$ is assumed to be low permeability zone. Any location which contains sand was assigned the maximum $K_{h}$ of $11500 \mathrm{md}$, while any low permeability zone was assigned $K_{h}$ of $115 \mathrm{md}$. Throughout the model, every point had a constant ratio $K_{\imath} / K_{h}$ of 0.3 . As a result, this model had permeability contrast of $100 \%$. 
Figure 3. Two-phase permeability field for dual porosity reservoir with (a) 30\% (denoted $P_{30}$ ), and (b) $60 \%$ (denoted $P_{60}$ ) proportion of the low permeability zones

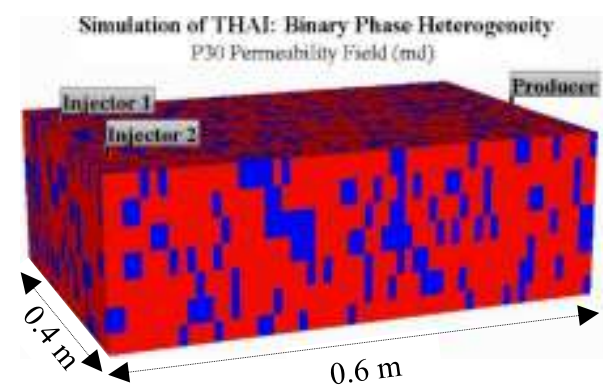

(a)

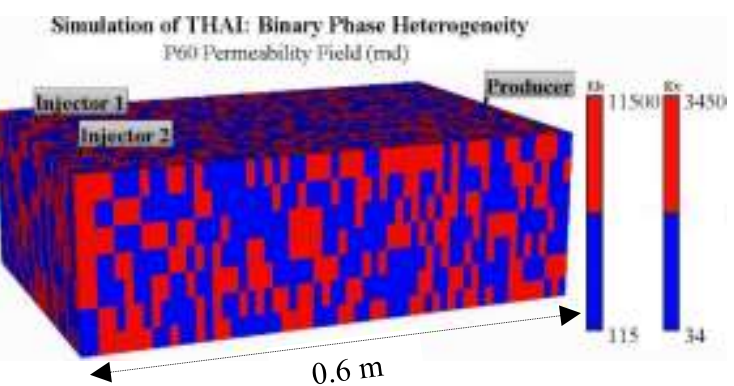

(b)

Another binary phase model is shown in Figure 3(b) in which the reservoir was highly populated by low permeability zones. In this case, the probability $\left(P_{60}\right)$ of finding low permeability zones at any given location was at most $60 \%$. The discontinuous low and high permeability zones were assigned $K_{h}$ values of 115 and $11500 \mathrm{md}$, respectively. Again, throughout the reservoir, the ratio $\mathrm{K}_{\mathrm{v}} / \mathrm{K}_{\mathrm{h}}=0.3$ was kept constant. This model also had a permeability contrast of $100 \%$.

\subsubsection{Reservoir with inter-bedded shale layers}

In order to study the impact of shales on the THAI process, a generic reservoir model was used. In this study, the same reservoir model as in Figure 3(a) was used except that the blue phase (i.e. the low permeability zones) was replaced by shales. The shales were assumed to have both zero horizontal and zero vertical permeability. Therefore, STARS assumed the shaly zones to have zero porosity and hence be free of fluid (CMG, 2012). The absolute horizontal permeability of the higher permeability (red) phase was kept the same (i.e. $11500 \mathrm{md}$ ) and the ratio $K_{\mathrm{v}} / K_{h}=0.3$ was kept constant throughout the reservoir. 


\subsubsection{Layered reservoir}

The impact of gradation in reservoir permeability on the THAI process was studied using a reservoir model with the same dimensions as the base case homogeneous model. Only one study in which the effect of a layered reservoir on in-situ combustion has been reported (Akkutlu and Yortsos, 2005). In order to properly represent the gradual decrease in both the permeability and porosity, the following permeability-porosity relationship was used:

$$
K=K_{o} e^{\left[f\left(\frac{\varphi-\varphi_{\mathrm{o}}}{1-\varphi_{\mathrm{o}}}\right)\right]}
$$

where $K_{o}$ and $\varphi_{\mathrm{o}}$ are the initial permeability and porosity, respectively. $K$ and $\varphi$ are the current permeability and porosity, respectively, and $f$ is a multiplication factor (Li et al., 2015, 2016). The multiplication factor is calculated by using shale absolute horizontal permeability and porosity of $1 \mathrm{md}$ and $1 \%$ respectively as reference values (Le Ravalec et al., 2009). In combination with the base case permeability and porosity, the $f$ value used in the current study is 18.71 . Depending on the original depositional environment and subsequent movements, in some oil reservoirs, the permeability and porosity could progressively decrease from the top to the base of the reservoir. In others, the reservoir could become more permeable, and thus (typically) porous, as the depth of burial is decreased. Another possibility is the progressive decrease or increase in permeability and thus porosity along a crosssection. As a consequence, four different reservoir models were modelled under the same operating conditions as the base case model. These were: (i) High Permeability Centre (HPC), in which the permeability and porosity progressively decrease away in successive planes beyond the vertical middle plane (Figure 4(a)), (ii) Low Permeability Centre (LPC) in which there is a progressive increase in both permeability and porosity in successive planes beyond the vertical middle plane (Figure 4(b)), (iii) Topdown Permeability Increase (TPI) modelled the effect of gradual increase in permeability and porosity from the top to the bottom of the reservoir (Figure 4(c)), and (iv) Top-down Permeability Decrease (TPD) involved the gradual decrease in permeability from the top to the base of the reservoir (Figure $4(d))$. 
Figure 4. Permeability Field in Layered Reservoir for (a) HPC, (b) LPC, (c) TPI, and (d) TPD Models

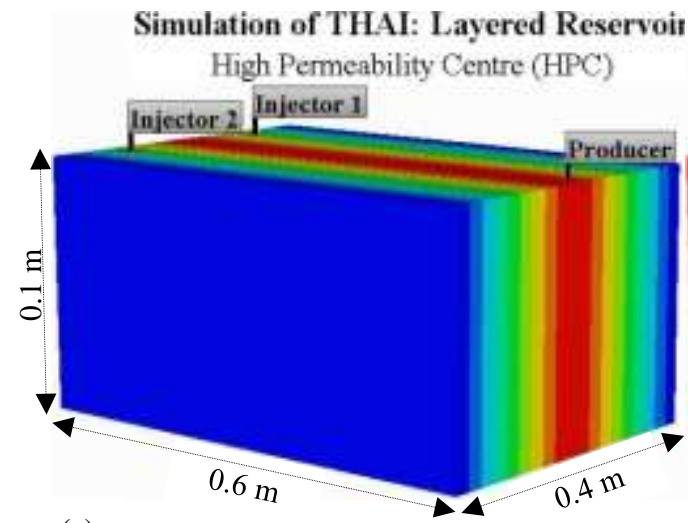

(a)

Simulation of THAI: Layered Reservoi Top-down Permeability Increase (TPI)

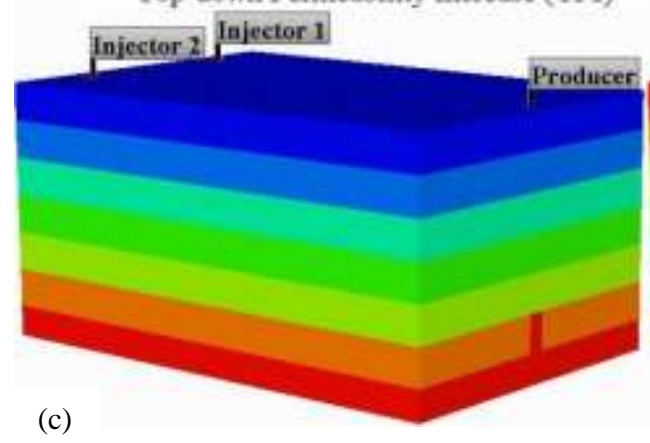

Simulation of THAI: Layered Reservoir Low Permeability Centre (LPC)

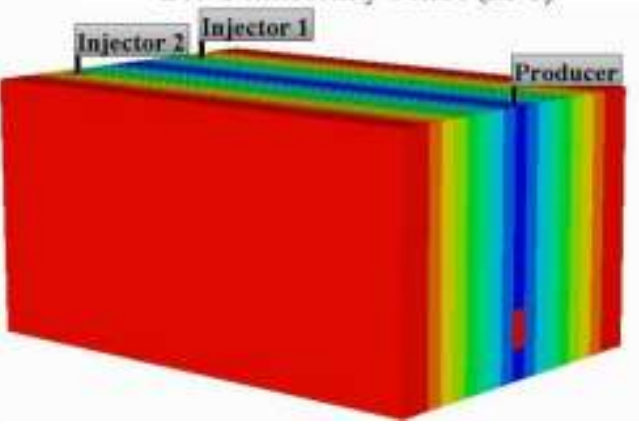

(b)

Simulation of THAI: Layered Reservoir Top-down Permeability Decrease (TPD)

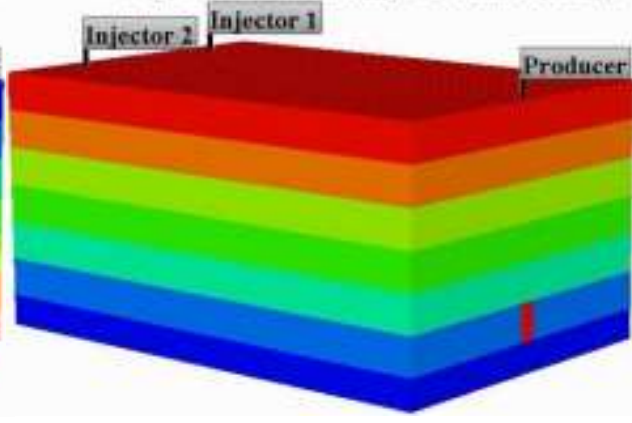

(d)

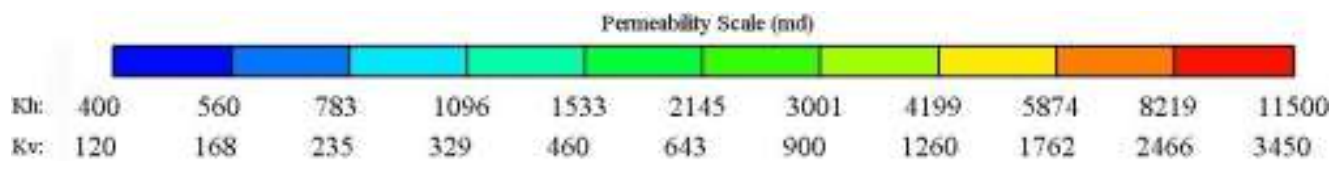

In all the four cases, the lowest absolute horizontal permeability was $402.5 \mathrm{md}$ and the corresponding porosity was $22.17 \%$. In the HPC and LPC models, the change of permeability between every two adjacent vertical planes was by a factor of $150 \%$, while in the TPI and TPD models the change in the permeability between every two adjacent horizontal planes was by a factor of $175 \%$. Also, throughout each of the models, the ratio $K_{\downarrow} / K_{h}=0.3$ was maintained. Irrespective of the absolute permeability assigned in general to horizontal layer six (see Figure 4), where the horizontal producer (HP) was located, the blocks around the HP were always assigned a horizontal absolute permeability of 11500 md. During the simulations of operation, each of these models was steam-preheated for 30 minutes 
prior to six hours of combustion period. This was to allow for the same quantity of heat to be supplied to the reservoir as in the experimental model.

\subsubsection{Limits to safe operation of THAI with permeable cap-rock}

The Athabasca oil sand, which is the largest of the three Canadian oil sand reserves, and is considered in the current study, is contained within the Lower Cretaceous McMurray formation, the oldest formation in the so-called Mannville group (Mossop, 1980; Petrobank, 2009; Deschamps et al., 2012). The McMurray formation divides into three units, with the highest concentration of tar sand being located in the middle unit. This is separated from the upper unit by a thin shale layer which is followed by very fine- to medium-grained estuarine bay-fill cross-bedded sand deposit with gradual upwardcoarsening. Directly overlaying the upper McMurray zone is the Wabiskaw sand which is the bottom part of the Clearwater formation and is made up of silty marine sand. Laying above this is the shale layer that provides the cap-rock which trapped the bitumen in place. This shale layer, also known as the lower Clearwater shale, together with the Wabiskaw sand, constitute the first sand-shale sequence of the Clearwater formation. The second sand-shale sequence is the Clearwater sandstone and the upper Clearwater Shale. The former is made up of a marine shore-face complex and contains a very large quantity of gas while the latter is composed of sandy siltstone and provides the seal for the underlying gas reservoir. The two sand-shale sequences of the Clearwater formation are overlain by the Grand Rapids formation which is the youngest member of the Mannville group (Mossop, 1980; Petrobank, 2009).

Petrobank (2009) observed, from the results of well logs, that there was a very small gas zone in the upper McMurray unit. They also reported that the bitumen cap-rock (i.e. the lower Clearwater shale), graded rapidly, in an upward fashion, into the Clearwater sandstone. This shows that in their project survey area, going vertically downward, two shale-gas sequences would be followed by shale-bitumen sequence. A quite similar observation, reported by Pooladi-Darvish and Mattar (2002), was made in 
another project site in the Athabasca region. Therefore, before any in-situ combustion heavy oil recovery technique is implemented, the effectiveness of a cap-rock, such as the lower Clearwater shale in providing the necessary sealing, separating the richest two hydrocarbon pools, must be investigated. This will involve establishing whether a direct hydraulic communication between the tar sand reservoir, and the overlaying Clearwater sandstone gas deposit, would matter. This consideration is very important for the deployment of the THAI process, where the sustenance, and thus the stability, of the combustion front is critical to the success of continuous oil production. Therefore, the injected air must be confined within the tar sand reservoir for two essential reasons: (i) the continuity of the process, so that enough air always reaches the combustion front to maintain economical air to oil ratio, and (ii) the prevention of auto-ignition of the overlying gas in the event where enough heat, from the bitumen combustion zone, is conducted, or convected, or both, to the gas reservoir.

Fluid migration into an overlying gas reservoir could potentially take place due to the presence of one, or a combination, of the following: (i) discontinuity in the cap-rock, (ii) some permeability in the caprock, and (iii) presence of fractures due to pressure build up and thermal stress. Where the cap-rock is continuous, such as the case in the Athabasca region (Pooladi-Darvish and Mattar, 2002; Petrobank, 2009), proper control of the fluid injection pressure to less than the shale fracture pressure could prevent the mass and heat convection transfer to the gas reservoir. The study of the effect of pressure build up and thermal stress on the integrity of the cap-rock requires geomechanical modelling and, consequently, is not considered here. This is because the STARS software does not allow discretised wellbore model option to be used in conjunction with the geo-mechanical simulation.

In this work, only the effect of the permeability of the cap-rock on confining the injection fluid in the bituminous reservoir was investigated at field scale. To accomplish this, five different models were developed for a generic tar sand reservoir section. For each model, the cap-rock was attached to the top of the oil reservoir, which has the same dimensions as the homogeneous reservoir model used in the 
development of a field-scale simulation for THAI in previous work (Rabiu Ado, 2017). The thickness of the cap-rock was assumed to be $50 \%$ of that of the oil reservoir (i.e. $12 \mathrm{~m}$ ), thereby leading to each model having an overall thickness of $36 \mathrm{~m}$ (as shown in Figure 5). The bitumen reservoir has the same oil properties and petrophysical data, in each model, as in previous work (Rabiu Ado, 2017). The caprock contains no oil and has very low absolute vertical permeability ranging from $10^{-6}$ to $10^{-3} \mathrm{md}$ (Pooladi-Darvish and Mattar, 2002; Chen, 2009). However, some authors have used a maximum permeability value of 0.001 md (Le Ravalec et al., 2009; Fatemi, 2012), and, in one case, a value of 0.1 md was used (Pooladi-Darvish and Mattar, 2002). The porosity and permeability of the cap-rock, used in each model, are shown in Table 1. In each case, it is assumed that only gas was present in the pore space of the cap-rock.

Figure 5. Simulation reservoir model containing cap-rock and bitumen reservoir

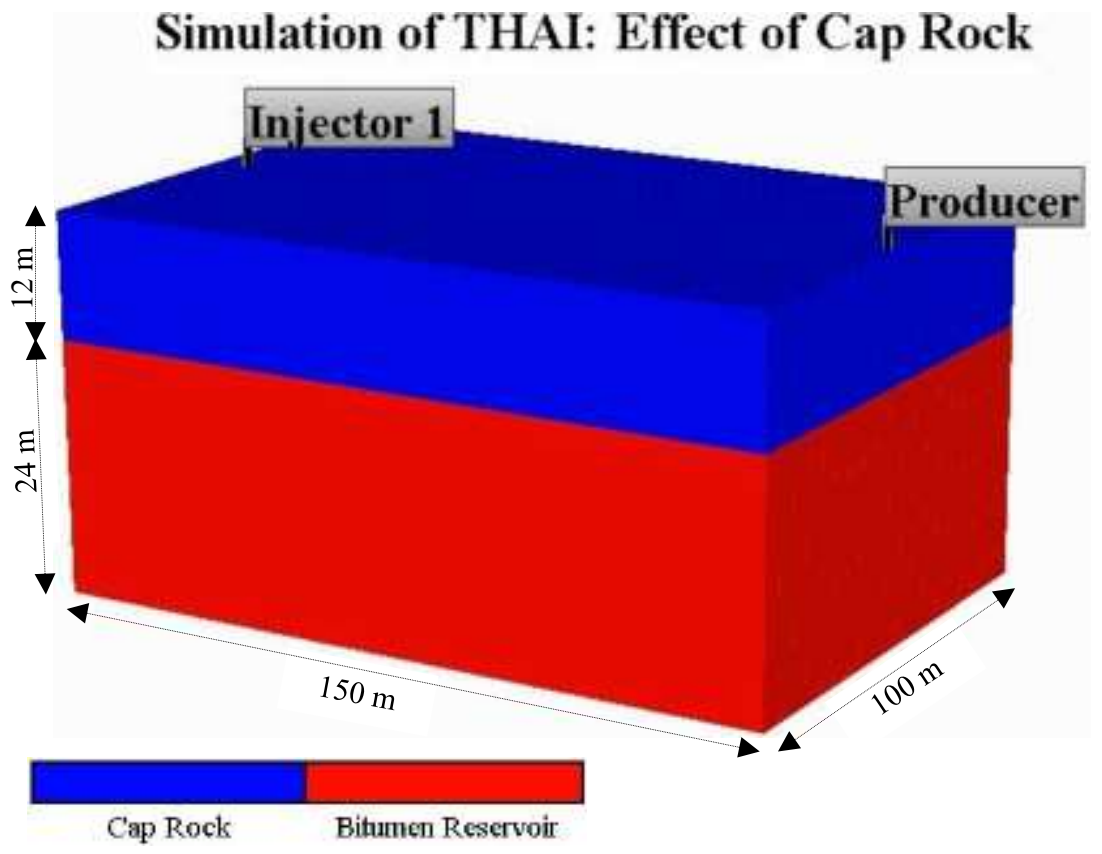

Each model has the vertical injector and the horizontal producer wells arranged in a direct line drive (DLD) configuration. In each model, steam was injected at a rate of $\approx 500 \mathrm{bbl} \mathrm{day}^{-1}$ (CWE) during the 
pre-ignition heating cycle (PIHC) for a period of 104 days. Air injection rate was $20000 \mathrm{Sm}^{3} \mathrm{day}^{-1}$ and was kept constant for 2 years. The horizontal producer (HP) was controlled using a bottom hole pressure of $2800 \mathrm{kPa}$, which is the typical reservoir pressure in the Athabasca region of Canada. All the models were run under exactly the same conditions in order to allow for comparison.

Table 1. Cap-rock porosity and permeability of reservoir models

\begin{tabular}{|l|l|l|l|l|}
\hline Model & Model Description & Porosity & $\mathbf{K}_{\mathbf{H}}(\mathbf{m d})$ & $\mathbf{K}_{\mathbf{V}}(\mathbf{m d})$ \\
\hline C1 & No cap-rock & - & - & - \\
\hline C2 & Contains cap-rock & 0.01 & 1 & 0.1 \\
\hline C3 & Contains cap-rock & 0.01 & 1 & 0.3 \\
\hline C4 & Contains cap-rock & 0.09 & 10 & 3.0 \\
\hline
\end{tabular}

\section{RESULTS AND DISCUSSION}

The results are presented in this section for each of the models in the order outlined above.

\subsection{Homogeneous model with constant $K_{\downarrow} / K_{h}$}

Figure 6 shows the \%OOIP recovery, CAOR, fuel availability, and average peak temperature as a function of horizontal absolute permeability with the $K_{\downarrow} / K_{h}=0.3$ at the end of $320 \mathrm{~min}$ of combustion. At high permeability, the ease with which the mobilised hot fluid flows means heat is easily distributed through the reservoir. This results in an increase in thermal cracking, causing more fuel deposition, as 
shown in Figure 6(c). In this work, the produced oil referred to the amount of oil that has already been recovered at the end of the 320 minutes of process time while the recoverable oil is the amount of oil left inside the reservoir at the end of the 320 minutes of process time and which can be recovered by extending the process time. In Figure 6a, it can be observed that the percent of recoverable oil decreases with the increase in absolute permeability. This is because, as the permeability increases, the percent of oil recovered increases and the fuel availability increases (Figure 6c). And that means, at higher permeability, less oil that can be recovered is left inside the combustion cell and hence the decrease of percent of recoverable oil. Also, the cumulative air-to-oil ratio decreases with increase in absolute permeability (Figure 6b) and this corresponds to the decrease in operating cost. Figure $6 \mathrm{~d}$ shows that the average peak temperature increased with the increase in absolute permeability over the range of 2875 to $7200 \mathrm{md}$. As the permeability was increased beyond $7200 \mathrm{md}$, the average peak temperature stabilized, implying insensitivity to further increase in the permeability. The increase in peak temperature can be attributed to the higher fuel concentration at the high permeability. At higher permeabilities, it was observed that oxygen production began earlier, despite the fact that more fuel was deposited, compared to when the permeability is low. This was because at high permeability, the combustion front reached the toe of the horizontal producer (HP) earlier than at low permeability. Overall, oxygen utilisation decreased with the increase in permeability. 
Figure 6. (a) \%OOIP recovery, (b) CAOR, (c) Fuel Availability, and (d) Average Peak Temperature as Function of Horizontal Absolute Permeability with the $K_{\downarrow} / K_{h}=0.3$ at the end of $320 \mathrm{~min}$ of combustion.

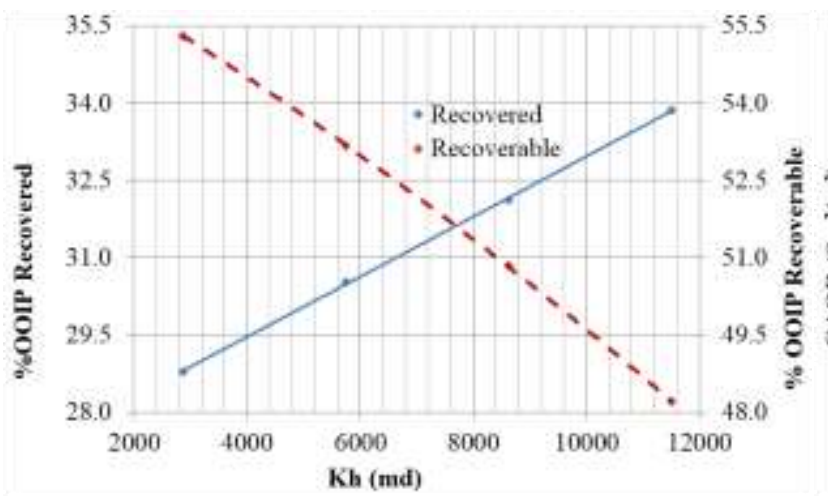

(a)

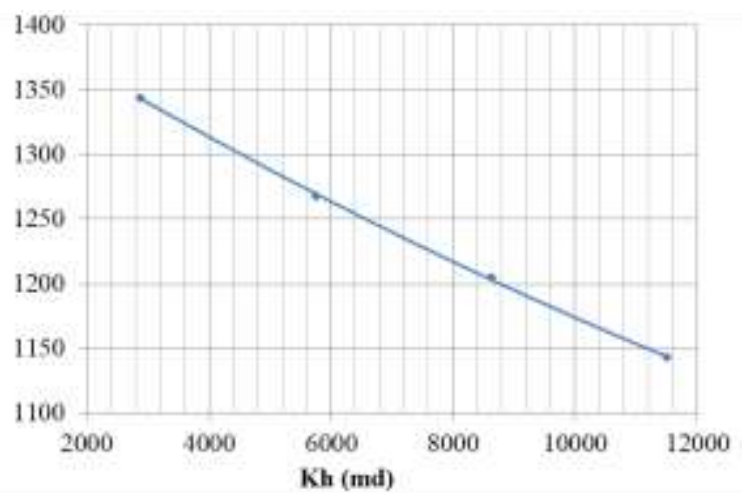

(b)

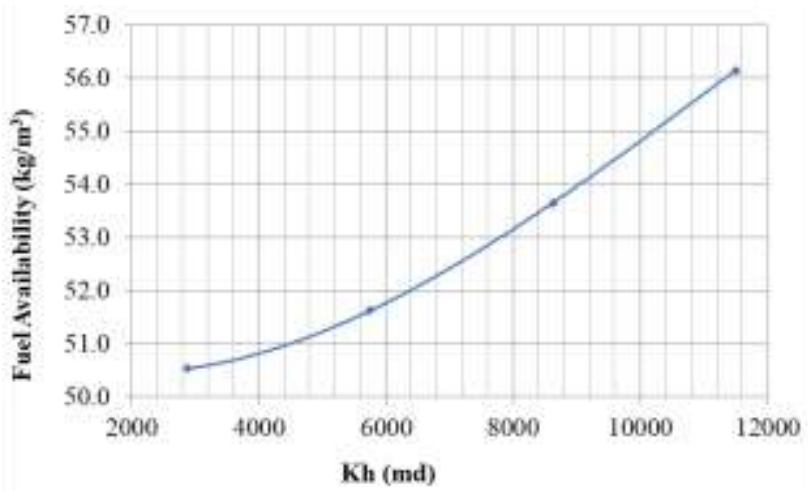

(c)

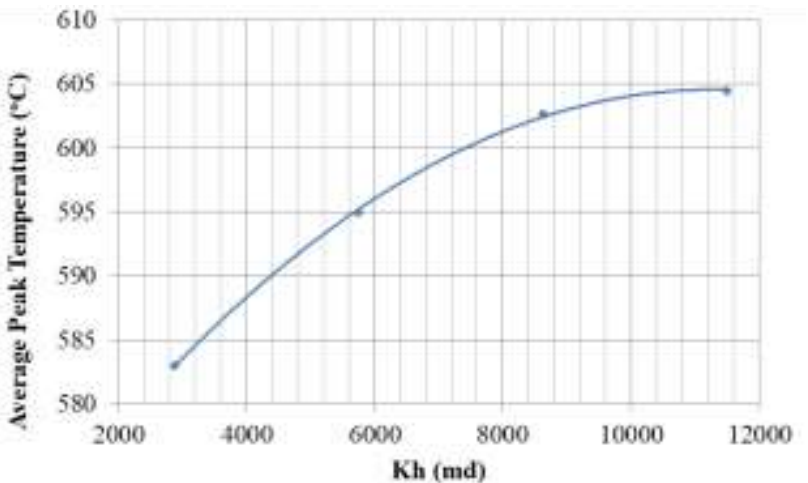

(d)

The distribution of oxygen in Figure 7 delineates the combustion front. From the shapes of the combustion front given in Figure 7(a), it can be seen that a higher percentage of the reservoir volume is swept by the combustion front, when the horizontal permeability is $11500 \mathrm{md}$, compared to when it is $2875 \mathrm{md}$ (decrease of $75 \%$ ). The same can be observed from the temperature distribution profile given in Figure 7(b). As can be seen in Figure 7(c), the high temperature zone (542 to $610{ }^{\circ} \mathrm{C}$ ) is closer to the mobile oil zone (MOZ) when the permeability is higher compared to when the permeability is reduced by $75 \%$. The concentration of the oil flux vectors (superimposed on Figures 7(b) and 7(c)) immediately above the HP is lower at higher permeability compared to when the permeability is $75 \%$ smaller. In contrast, below the HP, the MOZ is densely populated with oil flux vectors at higher 
permeabilities. This is because the mobilised oil easily reaches the bottom horizontal plane, from the adjacent vertical planes, before it flows upward into the HP. A further effect, which the decrease in permeability has on the process, is related to the areal or volumetric zone, ahead of the combustion front, occupied by higher oil saturation $(88-100 \%)$. The expanded oil banking zone, at the higher permeabilities, is also due to the ease with which the fluids flow horizontally, thereby mixing with the unaffected heavy oil in the cold oil zone. However, overall, it is noticeable that the nature of the oil drainage, as well as heat distribution, is quite similar. Interestingly, also, the position of the MOZ in the sandpack, as indicated by the largest oil flux vectors, indicates that the temperature is in the range of $150{ }^{\circ} \mathrm{C}$ to nearly $300{ }^{\circ} \mathrm{C}$, regardless of the value of the permeability used. 
Figure 7. Combustion front shape (a), temperature profile (b), and oil saturation profile (c) in the homogeneous sandpack, for (i) $K_{h}=11500 \mathrm{md}$ and (ii) $K_{h}=2875 \mathrm{md}$ at 320 minutes.

(a)
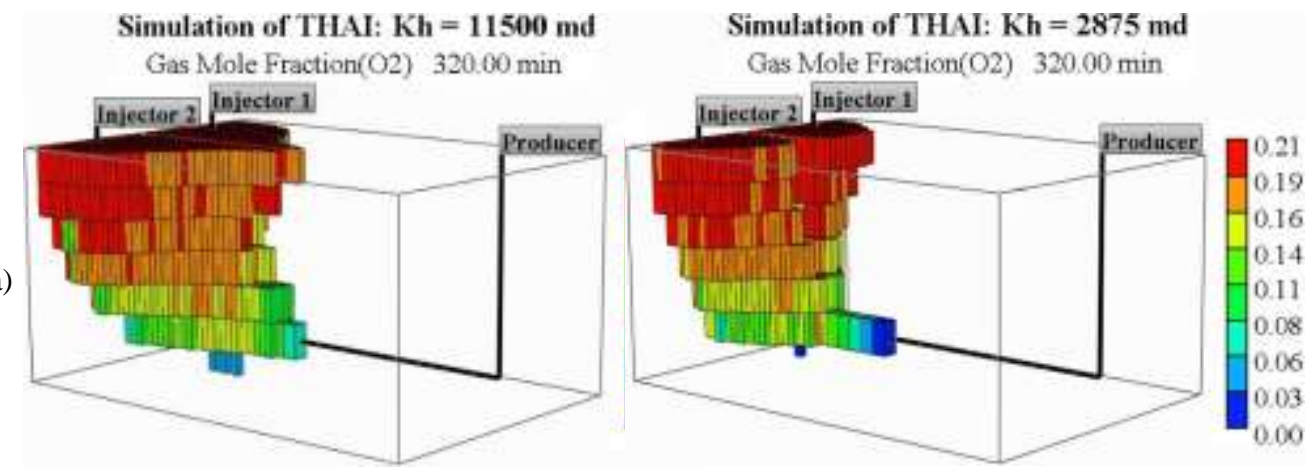

(i)

3D Shape of combustion front

(ii)
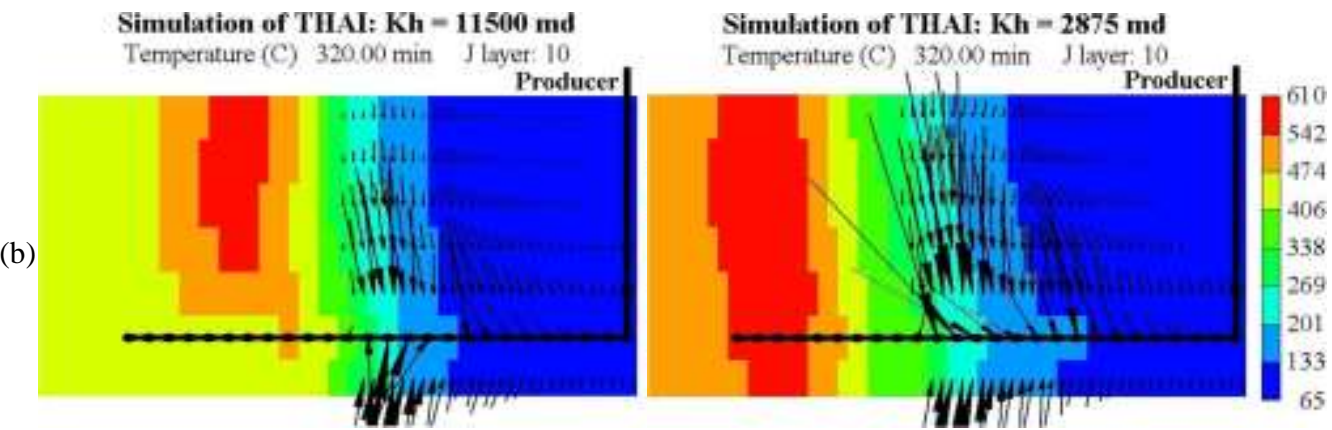

(i)

Temperature profiles along the vertical mid-plane

(ii)

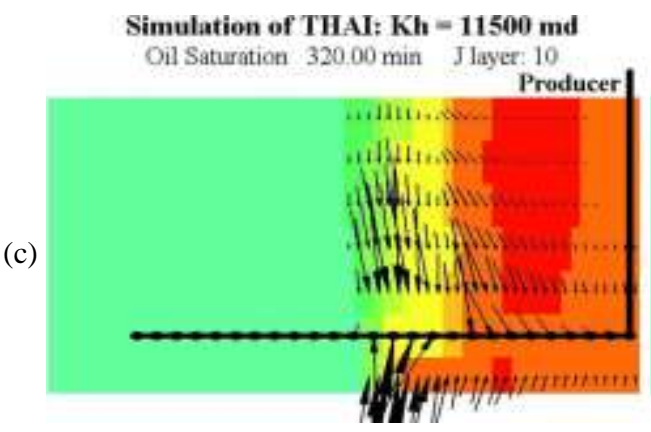

(i)

Oil saturation profiles along the vertical mid-plane

Simulation of THAI: $\mathbf{K h}=\mathbf{2 8 7 5} \mathrm{md}$

Oil Saturation $320.00 \mathrm{~min}$ Jlayer 10

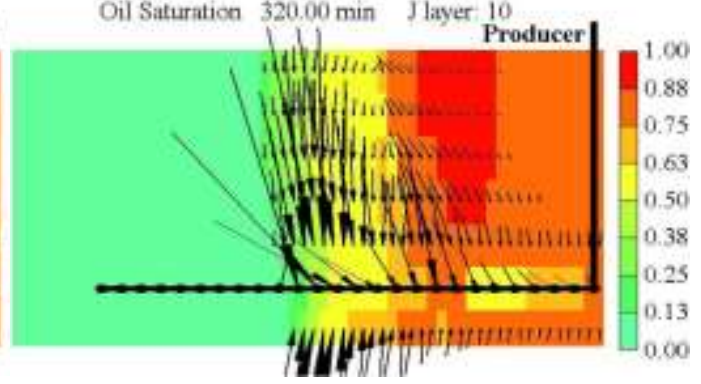

(ii)

\subsection{Random reservoir heterogeneity}

Figure 8 shows the shape of the combustion zone at 320 minutes for a reservoir model with random heterogeneity. It can be observed that the volume of the reservoir swept by the combustion front, as shown approximately by the oxygen profile, was smaller than when the absolute permeability was homogeneously 11500 md (see Figure 7(a.i)), but is bigger than when the permeability was homogeneously reduced to $2875 \mathrm{md}$ (see Figure 7(a.ii)). In both the homogeneous (Figure 7(a)) and the 
heterogeneous (Figure 8(a)) models, the 3D shape of the combustion front, in the radial direction away from the HP, is forward leaning, which is one of the indicators of stability. However, there is an increased penetration of oxygen along the HP, which shows that the process is tending to partial instability.

Figure 8. Combustion front profiles in three-dimensions (a), and along the vertical mid-plane (b) for simulation of THAI in a reservoir model with random heterogeneity after 320 minutes.

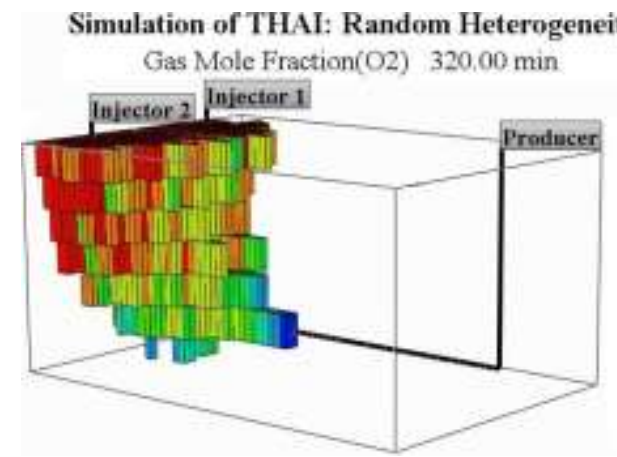

(a)

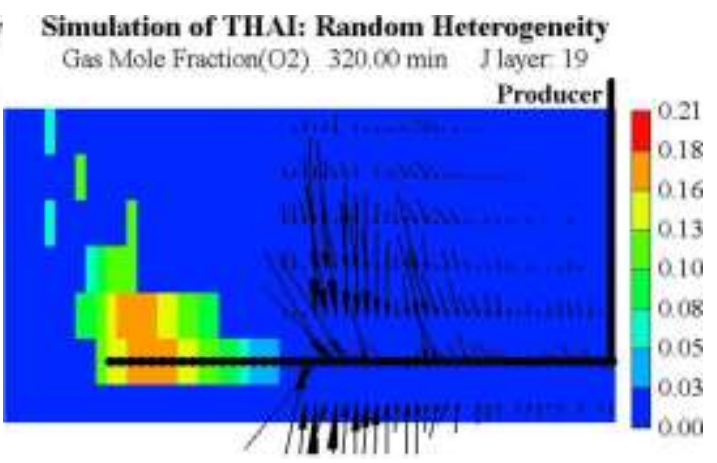

(b)

The type of heterogeneity affects the pattern of oxygen utilisation. Along the vertical mid-plane, the oxygen distribution, from the top of the reservoir to the toe of the HP, is discontinuous. However, due to the uniform combustion front propagation from the top to the base of the reservoir along the adjacent vertical planes, a large oxygen-rich zone is still formed around the toe of the HP in the mid-plane. While a similarly-sized oxygen-rich zone was also observed for the homogeneous models, in contrast, they did not exhibit isolated oxygen zones, around the top of the reservoir, as in the heterogeneous models.

For the purpose of comparison, Figures 9(a)-(c) part (i) show the shape of the oxygen profile at the top horizontal plane for two homogeneous (11500 and $5750 \mathrm{md})$ models, and the heterogeneous model. Unlike the homogeneous case (Figure 9(a.i) \& Figure 9(b.i)), the shape of the combustion front, for the heterogeneous case, is irregular and distorted (Figure 9(c.i)). This is due to the variability of the fuel 
concentration ahead of the combustion front. It means that the process will be difficult to control, where the combustion front progressively advances towards, and along, the low fuel concentration zone, thereby forming oxygen fingers. Once the combustion front reaches the highest permeability zones, which directly connect to the HP, oxygen breakthrough could occur. This would lead to unsafe or uneconomical operation. However, these possibilities were not observed in this current study, since the oxygen utilisation efficiency was $95.4 \%$ for the homogeneous high permeability reservoir, and $95.1 \%$ for the heterogeneous reservoir. The slight decrease in the oxygen utilisation can be explained by the difference in the average fuel availability, given the average fuel availability for the $11500 \mathrm{md}$ homogeneously permeable reservoir was $56.1 \mathrm{~kg} \mathrm{~m}^{-3}$, which was $3.9 \mathrm{~kg} \mathrm{~m}^{-3}$ higher than that for the heterogeneous reservoir.

The oil saturation profiles show that the areal displacement of oil is higher for models with higher permeability, and decreased with a decrease in permeability (Figure 9(a.ii) \& Figure 9(b.ii)). The oil flow pattern in the heterogeneous reservoir was quite tortuous (Figure 9(c.ii)). This probably explains why the oil recovery, after 320 minutes of combustion, was $29.8 \%$ OOIP for the heterogeneous model, which was $4.1 \%$ OOIP, and $0.8 \%$ OOIP, lower than for the homogeneous reservoirs with horizontal permeabilities of $11500 \mathrm{md}$, and $5750 \mathrm{md}$, respectively. 
Figure 9. The oxygen profile (i) and oil saturation (ii) at the top horizontal plane for two homogeneous (11500 (a) and 5750 md (b)) models, and the heterogeneous model (c), after 320 minutes.

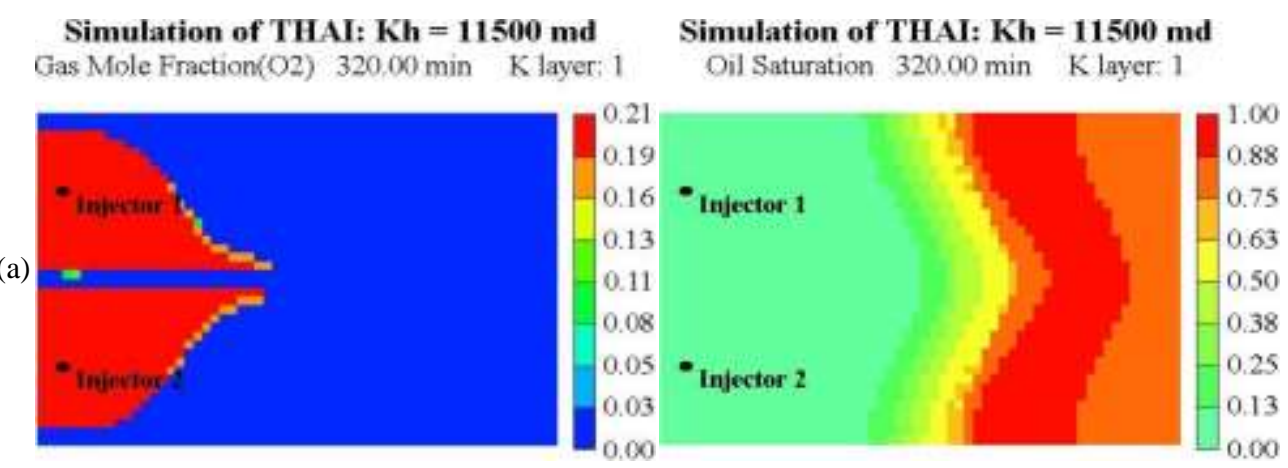

(i) Homogeneous $\left(\mathrm{K}_{\mathrm{h}}=11500 \mathrm{md}\right.$ and $\left.\mathrm{K}_{\mathrm{v}} / \mathrm{K}_{\mathrm{h}}=0.3\right)$

(ii)

Simulation of THAI: $\mathbf{K h}=\mathbf{5 7 5 0} \mathbf{m d} \quad$ Simulation of THAI: $\mathrm{Kh}=\mathbf{5 7 5 0} \mathbf{m d}$ Gas Mole Fraction $(\mathrm{O} 2) \quad 320.00 \mathrm{~min}$ K layer: 1 Oil Saturation $320.00 \mathrm{~min}$ K layer: 1

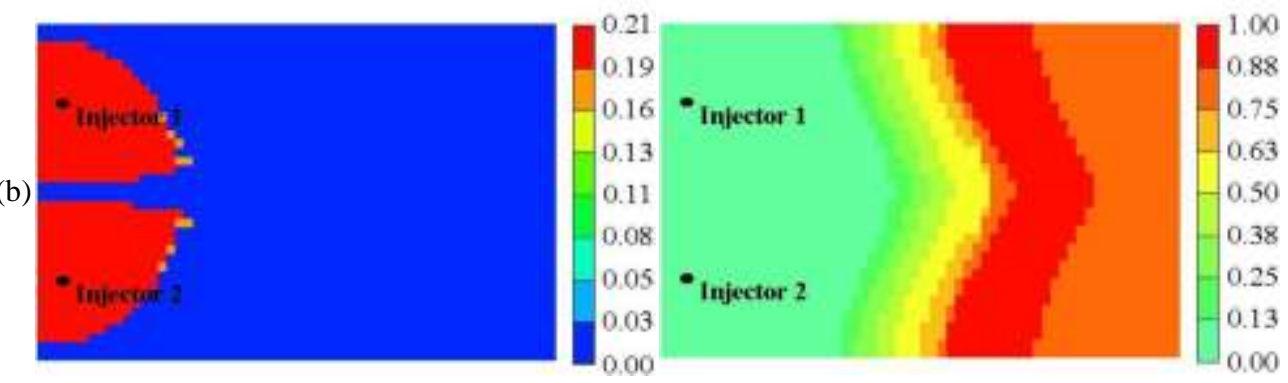

(i) Homogeneous $\left(\mathrm{K}_{\mathrm{h}}=5750 \mathrm{md}\right.$ and $\left.\mathrm{K}_{\mathrm{v}} / \mathrm{K}_{\mathrm{h}}=0.3\right) \quad$ (ii)

Simulation of THAI: Random Heterogeneity Simulation of THAI: Random Heterogeneity Gas Mole Fraction(O2) $320.00 \mathrm{~min}$ K layer; 1 Oil Saturation 320.00 min K layer: 1

(c)

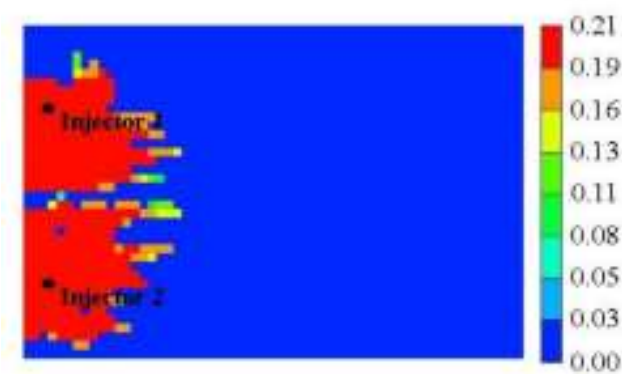

(i) Random Heterogeneity $\left(\mathrm{K}_{\mathrm{v}} / \mathrm{K}_{\mathrm{h}}=0.3\right)$

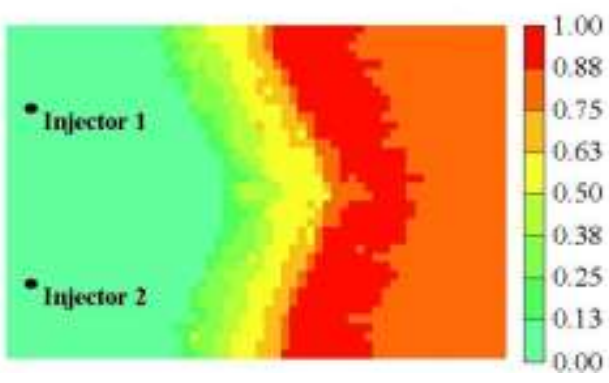

(ii)

\subsection{Dual porosity reservoir}

The larger portion of the reservoir swept by the $\mathrm{P}_{30}$ model, given in Figure 10(a), compared to that predicted for the $\mathrm{P}_{60}$ model, as shown in Figure 10(b), is because of the decline in the mobilised fluid flows with an increase in the probability of having low permeability zones. The models also show that 
the shape of the combustion front was determined by the predominant rock characteristics (i.e. low or high permeability). When the predominant rock characteristic was highly permeable (i.e. model $\mathrm{P}_{30}$ ), the combustion front preferentially propagated via the high permeability zones, thereby bypassing the low permeability regions (see Figure 10(c)). In contrast, when the reservoir was predominantly populated by low permeability zones (i.e. as in model $\mathrm{P}_{60}$ ), the combustion front became less channelled, and propagated evenly via the low permeability zones (see Figure 10(d)). Figure 10(e) shows that, for model $\mathrm{P}_{30}$, both at the top, and along the HP near the base, of the reservoir, the combustion front had the same velocity. However, in model $\mathrm{P}_{60}$, the rate of advance of the combustion front along the HP was higher than at the top of the reservoir (see Figure 10(f)). Figure 10(f) shows that the combustion front is then backward leaning. The wedge-like protrusion of the combustion front into the HP, predicted for model $\mathrm{P}_{60}$, is caused by the continuity of the high permeability zones along the HP. Since the speed of the combustion front is controlled by the dominant permeability rock characteristic, the presence of a continuous high permeability zone amidst a large proportion of low permeability zones would cause the combustion front to become channelized. It, therefore, follows that oxygen production would increase with the increase in the probability of finding low permeability zones.

A comparison of the plots of oxygen mole fraction as a function of combustion time for the two different models (as shown in Figure 10) shows that oxygen breaks through earlier when the reservoir contains a larger proportion of low permeability zones. The oxygen utilisation efficiency at the end of the 320 minutes combustion period for model $\mathrm{P}_{60}$ was $89.1 \%$, which was lower than for model $\mathrm{P}_{30}$ by $5.8 \%$. 
Figure 10. The shape of the combustion front for (a) the $\mathrm{P}_{30}$ model, and (b) the $\mathrm{P}_{60}$ model. Also shown are the oxygen concentration profiles along the top horizontal layer for (c) the $\mathrm{P}_{30}$ model and (d) the $\mathrm{P}_{60}$ model, and the oxygen concentration profiles along the vertical mid-plane for (e) $\mathrm{P}_{30}$ model and (f) the $\mathrm{P}_{60}$ model at the end of 320 minutes of combustion.

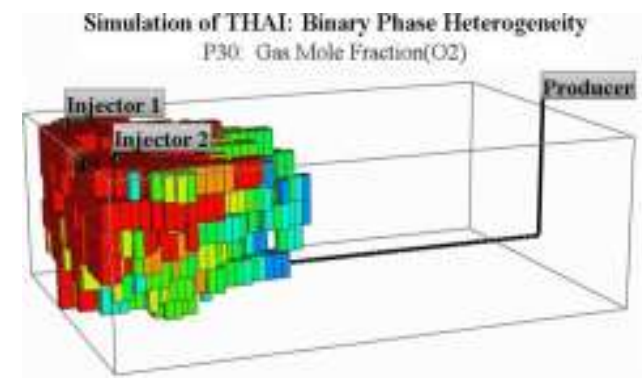

(a)

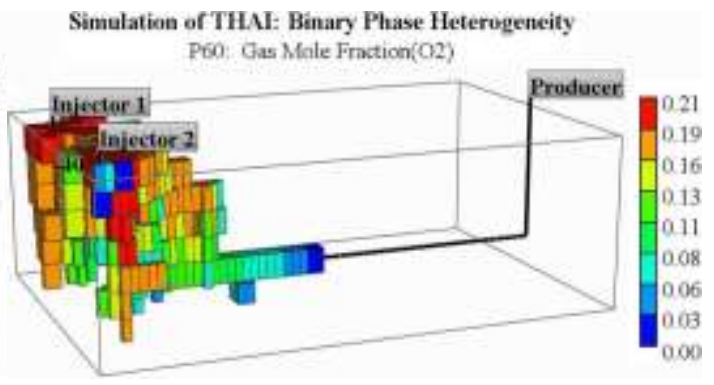

(b)

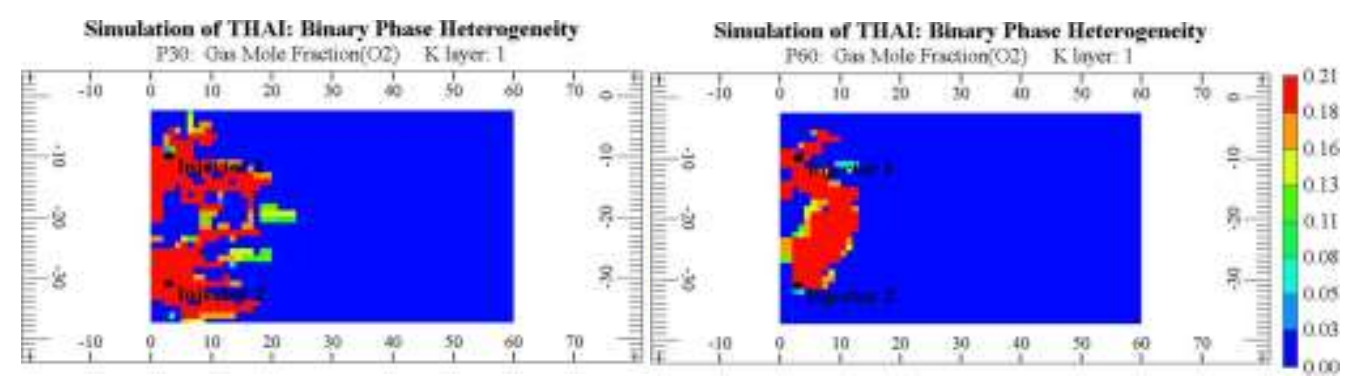

(c)

(d)

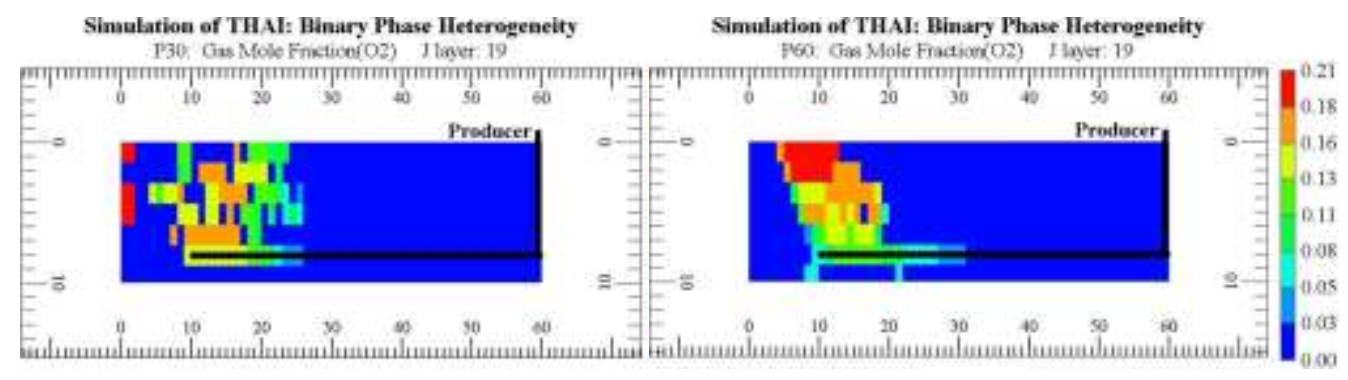

(e)

(f)

The oil flux vectors superimposed on Figure 11(a) show an ordered uniform oil drainage pattern for model $\mathrm{P}_{30}$ compared to when the proportion of the low permeability zones is increased for model $\mathrm{P}_{60}$ (see Figure 11(b)). In addition, the flow pattern for model $\mathrm{P}_{60}$ is asymmetrical despite the use of two vertical injectors. It can be observed from Figure 11(b) that there is still a significant amount of oil to recover around "Injector 2". The oil recovery at the end of the 320 minutes of combustion was 23 $\%$ OOIP for model $\mathrm{P}_{60}$, which was $6.3 \%$ OOIP lower than that for model $\mathrm{P}_{30}$. It therefore follows that 
assuming an element of symmetry during reservoir simulation could significantly result in overestimation of the oil production. Therefore, in order to obtain accurate simulation results, the full pattern of heterogeneity should be simulated. This result also shows that the performance of the THAI process is affected by the proportion of low permeability zones present in the production zone. This is because as the percentage of the low permeability zones is increased, say from $\mathrm{P}_{30}$ to $\mathrm{P}_{60}$, the advance, and hence bulldozing effect, of the combustion front is restricted. This, in turn, limits the volume of oil mobilised and drained into the producer. In addition, the average peak temperature was observed to be higher for model $\mathrm{P}_{30}$ (see Figure 11(c)) compared to in the case of model $\mathrm{P}_{60}$ (see Figure 11(d)). This could be attributed to the higher oxygen utilisation and the fact that more fuel was available in the case of model $\mathrm{P}_{30}$ (i.e. $57.7 \mathrm{~kg} \mathrm{~m}^{-3}$ which is $15 \mathrm{~kg} \mathrm{~m}^{-3}$ greater than that generated in the case of model $\mathrm{P}_{60}$ ).

Figure 11. Oil saturation profiles along the top horizontal plane for dual porosity model $\mathrm{P}_{30}(\mathrm{a})$, and model $\mathrm{P}_{60}(\mathrm{~b})$. Also shown are the temperature distribution profiles along the vertical mid-plane for (c) model $\mathrm{P}_{30}$, and (d) model $\mathrm{P}_{60}$ at the end of 320 minutes of combustion.

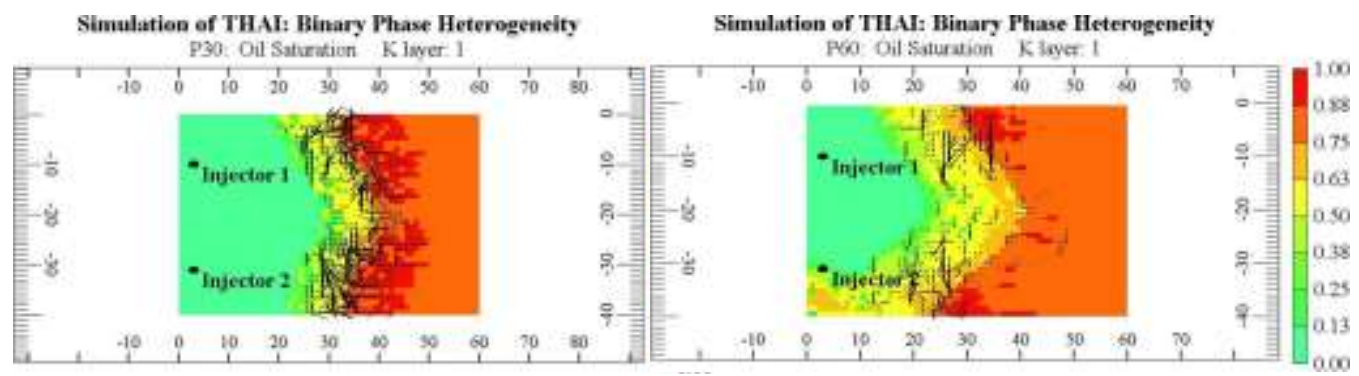

(a)

(b)

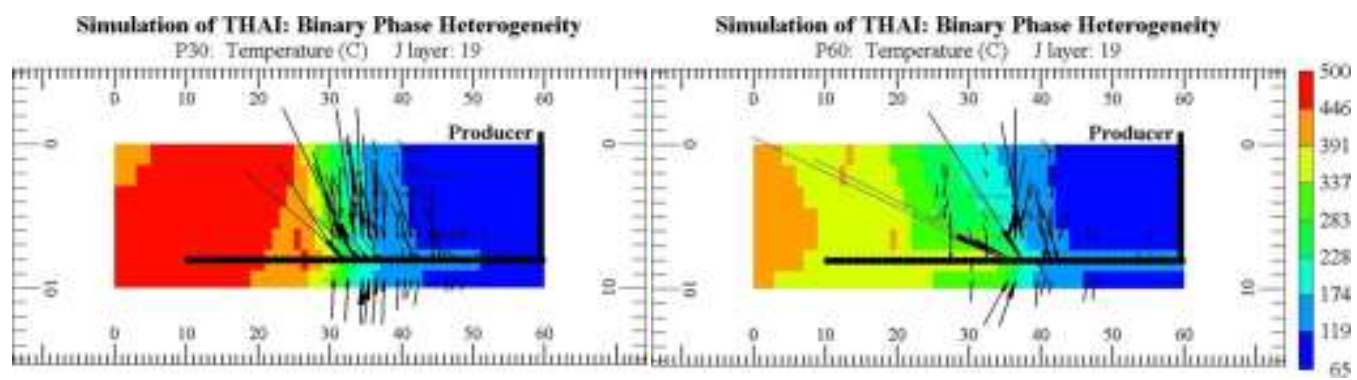

(c)

(d) 


\subsection{Reservoir with inter-bedded shale layers}

The permeability field of the model was such that the probability $\left(\mathrm{PS}_{30}\right)$ of finding shale at any given point was taken to be at most $30 \%$. As a result, the reservoir model had the same form as that shown in Figure 3(a) with the exception that the low permeability zones were now shaly zones.

For simulations of the THAI process, the oxygen concentration profile for the shaly reservoir $\left(\mathrm{PS}_{30}\right)$ was quite similar to that for the $\mathrm{P}_{30}$ model, as shown in Figure 10(c). The oxygen concentration profile was fairly tortuous, since the combustion propagated only through the permeable zones (as seen in Figure 12(a)). However, as the combustion was forced to propagate through the permeable zone only, it had to travel extra distance in all directions in order to sweep the equivalent oil reservoir volume to the case of model $\mathrm{P}_{30}$. That is why, along the vertical middle plane, at the end of the 320 minutes of combustion, the combustion front had covered $30 \mathrm{~cm}$ (in Figure 12(d)), which was $5 \mathrm{~cm}$ further than the corresponding distance it had covered for model $\mathrm{P}_{30}$ (as shown in Figure 10(e)).

The presence of impermeable zones resulted in the decrease in the overall oxygen utilisation from 94.9 $\%$, in the presence of low permeability zones, to $94.0 \%$ in the presence of shales. However, there was no evidence suggesting oxygen breakthrough due to preferential channelling into the production well. For the same realisation, fuel availability decreased from $57.7 \mathrm{~kg} \mathrm{~m}^{-3}$ in $\mathrm{P}_{30}$ to $52.0 \mathrm{~kg} \mathrm{~m}^{-3}$ when impermeable zones are present. This could explain why there is a slight difference in the oxygen utilisation.

From Figure 12(b), it can be seen that the presence of shales results in an asymmetrical temperature distribution. The combustion preferentially propagates through the area less populated by the presence of shales. Comparing the temperature distributions along the vertical middle plane for dual porosity and shaly reservoirs (in Figures 11(c) and 12(e)), it can be observed that presence of shale results in a 
decrease in the peak temperature. This is due to the higher specific heat capacity of rock compared to that of oil. This temperature difference will affect the rate of the cracking reactions.

Figure 12. Profiles along the top horizontal plane of (a) oxygen concentration, (b) temperature, and (c) oil saturation, and profiles along the vertical mid-plane of (d) oxygen concentration, (e) temperature, and (f) oil saturation, during simulations of THAI on the $\mathrm{PS}_{30}$ shaly reservoir model. The white phase in the oxygen and oil saturation profiles represent the blocks occupied solely by impermeable shales.

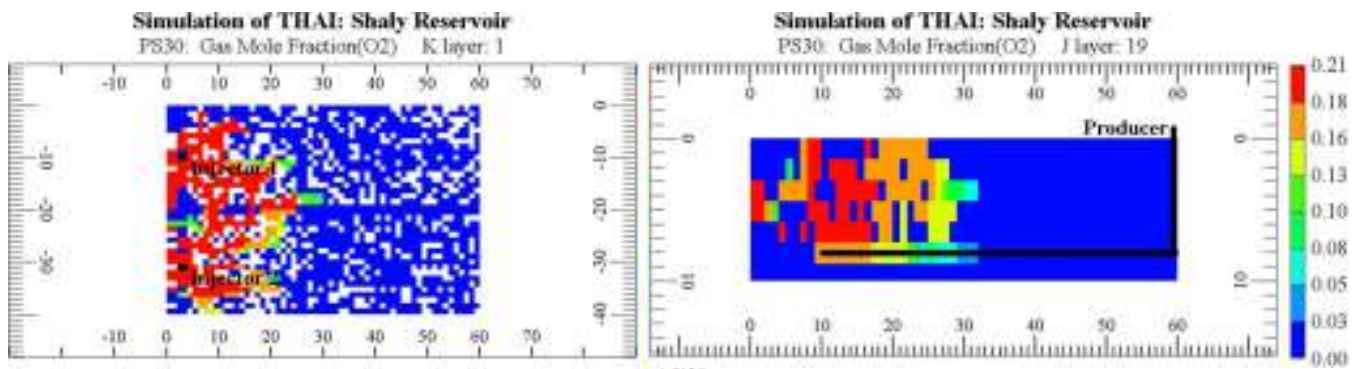

(a)

(d)

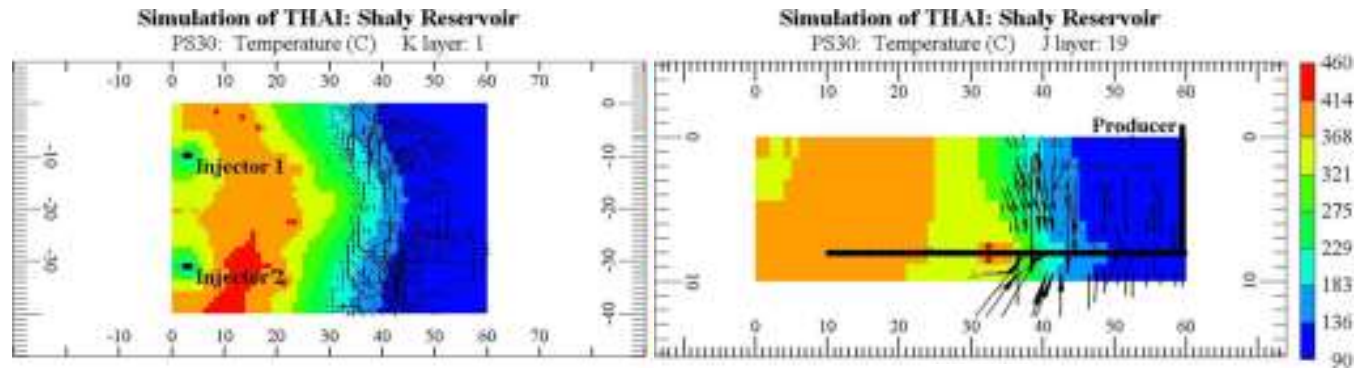

(b)

(e)

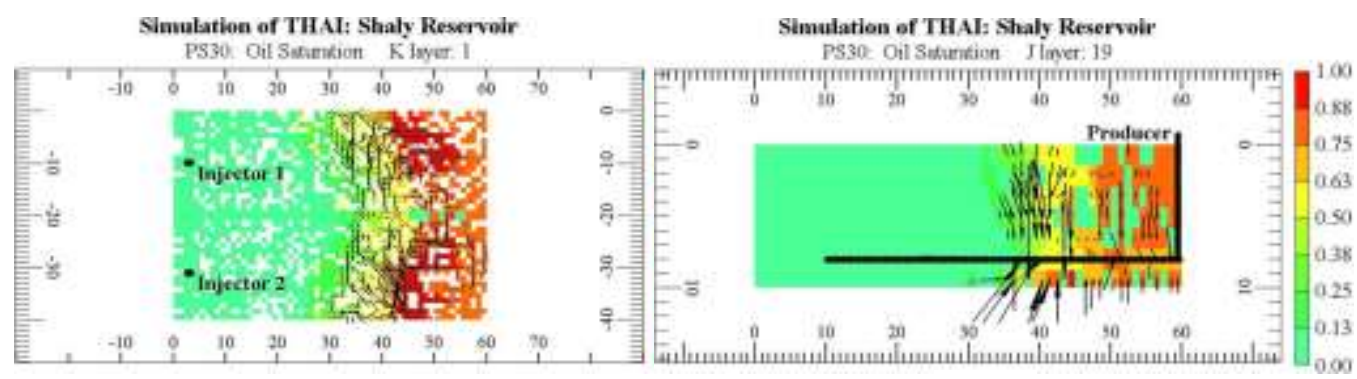

(c)

(f)

From Figures 11(a) and 12(c), it can be seen that the oil saturation profiles along the top horizontal plane are quite similar except that, when the shale was introduced, a higher volume of the reservoir was swept for the same air injection. Also, in the case of the shaly reservoir, there are closed zones where 
the trapped oil is irrecoverable even though the oil is heated by conduction. As a result, the oil in these zones is coked. This will significantly reduce the quantity of oil that will be cumulatively recovered.

A discontinuous shale distribution in the reservoir requires a larger air to oil ratio. The oil recovery at the end of the 320 minutes of combustion for the shaly reservoir was $38.7 \%$ OOIP, which was 9.5 $\%$ OOIP higher than achieved for dual porosity model $\mathrm{P}_{30}$. Further, the presence of shale resulted in an increased percent oil recovery compared to the $33.4 \%$ OOIP realised in the base case experimental model, which was homogeneously permeable. However, for the same air injection, $1870 \mathrm{~cm}^{3}$ of oil was cumulatively recovered in the presence of shale, compared to $2015 \mathrm{~cm}^{3}$ recovered from the $\mathrm{P}_{30}$ model, and $2310 \mathrm{~cm}^{3}$ recovered in the base case.

\subsection{Layered reservoir}

The presence of layers in the reservoir has significant implications for production losses and safety arising from oxygen breakthrough. From Figure 13, it can be seen that the combustion front in the layered reservoir models was skewed such that it was always concentrated in the highest permeability zones. For the HPC model (in Figure 13(a)) the combustion advance rate was highest along the central axis, in a toe-to-heel manner, thereby forming a funnel-like shape with the narrow end towards the heel. This shows that implementing the THAI process with the wells arranged in an SLD arrangement, and the HP located in the highest permeability zone, would result in unstable operation. This is because the combustion zone was increasingly tending towards the heel of the HP, which, should oxygen breakthrough, could be economically costly both in terms of production lost and safety.

The key operational performance parameters for the layered models are shown in Table 2 . The relatively high oil recovery for the HPC model was due to the ease with which the mobilised oil flows to the producer well in this reservoir configuration. Figure 14(a) shows that most of the produced oil comes 
from around the central axis of the model, where the permeability is highest. Further, the HPC model showed no rich banked oil region (i.e. 80 - $100 \%$ oil saturation) ahead of the high mobile oil zone (MOZ). However, on either side of the central axis, where the permeability was lowest, there was still a significant quantity of oil left to be recovered (i.e. oil saturation of $25-60 \%$ ). Further, it is noted that THAI operation on the HPC model had the highest oxygen utilisation of $92.9 \%$ (see Table 2).

For the LPC layered reservoir model, the combustion front split along the central axis (i.e. toe-to-heel plane) and formed two nearly independent fronts, which can be broadly described as conical in shape. Each of these fronts propagated stably in the high permeability zones on either side of the central axis. However, around the toe, the combustion fronts recombined to form a wedge-like protrusion into the HP, where the front advanced at faster rate compared to other parts of the fronts, thereby giving rise to a tendency to partial instability (see Figure 13(b)). The distance covered by the combustion front into the HP in the simulation on the LPC model was $26 \mathrm{~cm}$, which was $20 \mathrm{~cm}$ lower than that covered in the HPC model. The findings in Figure 13 show that implementing the THAI process to recover oil from a reservoir of a form similar to the LPC model would provide safe operation since oxygen production is delayed. Further, no coke was consumed along the central axis, and directly above the HP, due to the restriction to the fluid flow constituted by the presence of the smallest permeability in the reservoir. 
Figure 13. Shape of the combustion front in simulations of THAI operation on layered reservoir models shown in Figure 4, (a) HPC, (b) LPC, (c) TPI, and (d) TPD, after six hours of combustion.

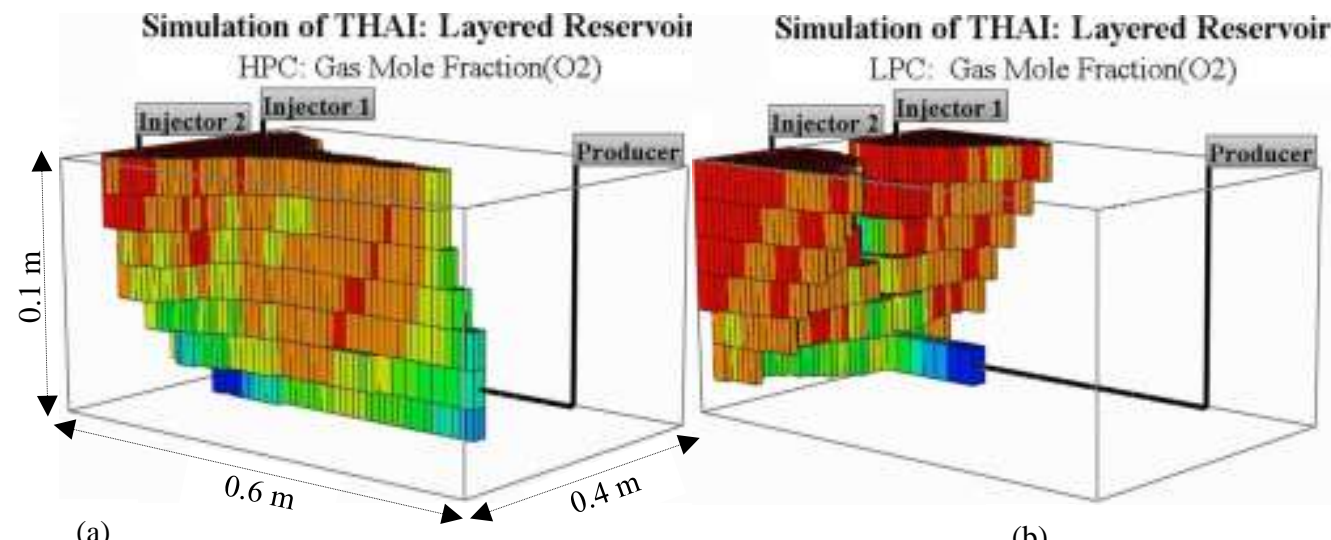

(a)

Simulation of THAI: Layered Reservoir TPL: Gas Mole Fraction(O2)

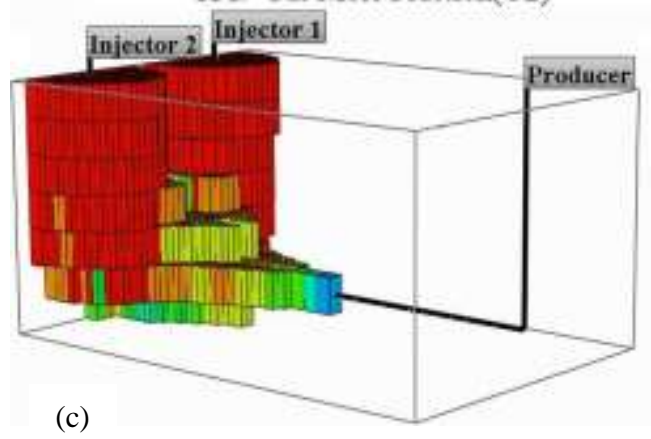

Simulation of THAI: Layered Reservoir TPD: Gas Mole Fraction(O2)

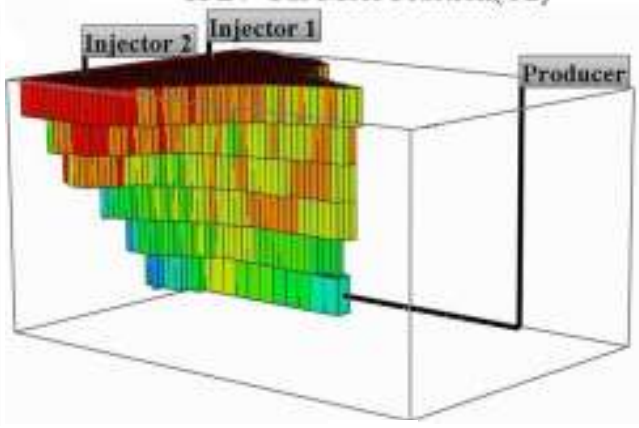

(d)

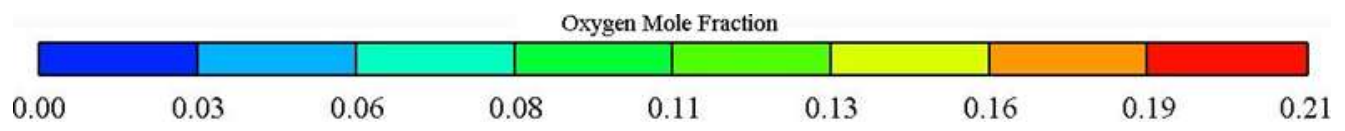


Table 2. Oil recovery, fuel availability, oxygen utilisation, and CAOR for simulations of THAI operation on layered reservoir models in Figure 4 after 6 hours of combustion

\begin{tabular}{|c|c|c|c|c|c|}
\hline Model & $\begin{array}{l}\% \text { OOIP } \\
\text { Produced }\end{array}$ & $\begin{array}{l}\% \text { OOIP } \\
\text { Recoverable }\end{array}$ & $\begin{array}{l}\text { Fuel } \quad A \\
\left(\mathrm{kgm}^{-3}\right)\end{array}$ & $\begin{array}{l}\% \mathrm{O}_{2} \\
\text { Utilisation }\end{array}$ & $\begin{array}{l}\mathrm{CAOR} \\
\left(\mathrm{Sm}^{3} \mathrm{~m}^{-3}\right)\end{array}$ \\
\hline HPC & 41.9 & 34.9 & 55.7 & 92.9 & 1427 \\
\hline LPC & 36.6 & 41.5 & 53.8 & 92.2 & 1594 \\
\hline TPI & 39.6 & 42.0 & 43.6 & 91.6 & 1524 \\
\hline TPD & 38.9 & 35.2 & 62.4 & 92.4 & 1532 \\
\hline
\end{tabular}

As can be seen from Table 2, for simulations on the LPC model, the oil recovered was lower than that recovered for simulations on the HPC model. This was due to the resistance, to the mobilised oil flow provided by the low permeability central axis, which has previously (Rabiu Ado et al., 2017) been shown to be the main pathway through which the mobilised oil, at experimental scale, reaches the HP. As seen in Figures 14(a)\&(b), unlike in the case of the HPC model, THAI simulations on the LPC model led to a large oil bank located ahead of the mobile oil zone (MOZ). In the simulations on the HPC model, the shape of the MOZ was parabolic, while in the case of the LPC model, it was aligned both horizontally and vertically. It follows that more of the mobilised oil could have been recovered, if the permeability of the central axis had been increased, while keeping the cumulative air injected the same. Similarly, the oxygen utilisation in the simulations on the LPC reservoir model was lower than that in the HPC reservoir by $0.7 \%$. This finding could be explained by the difference in fuel availability, since $1.9 \mathrm{~kg} \mathrm{~m}^{-3}$ less fuel was produced in the LPC model, compared to that in HPC (see Table 2). The higher \%OOIP recovered from the LPC reservoir than in the HPC reservoir (see Table 2) confirms that the main negative impact, that a low permeability along the central axis has on the THAI process, is the lowering of the quantity of mobilised oil reaching the producer well. 
Figure 14. Oil saturation profiles for simulations of THAI operation on layered reservoir models shown in Figure 4, (a) HPC, (b) LPC, (c) TPI, and (d) TPD, after six hours of combustion.

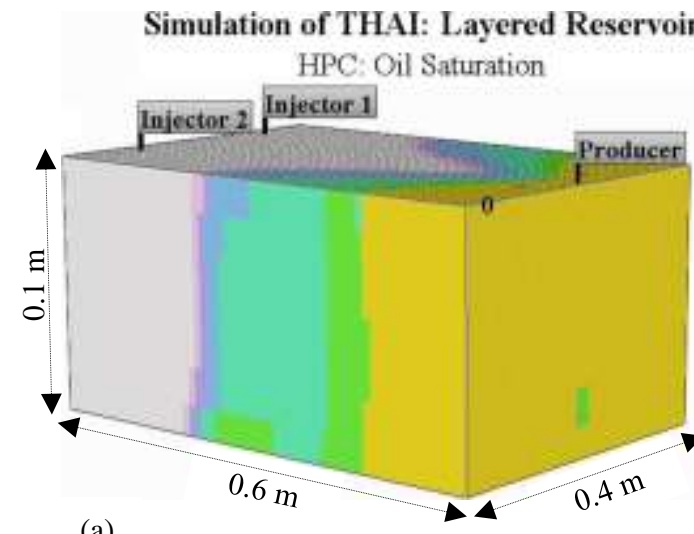

(a)

Simulation of THAI: Layered Reservoir TPI: Oil Saturation

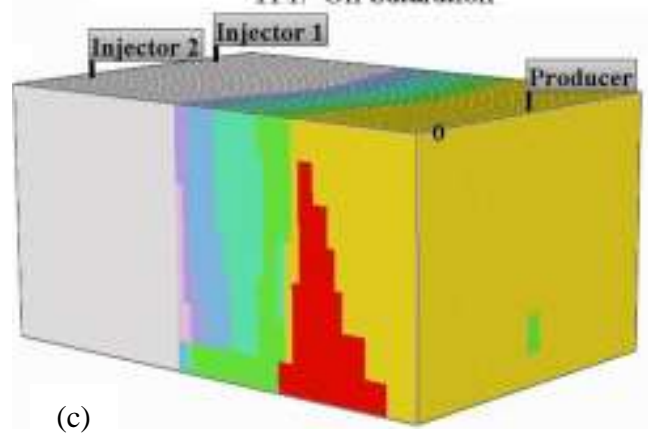

(c)

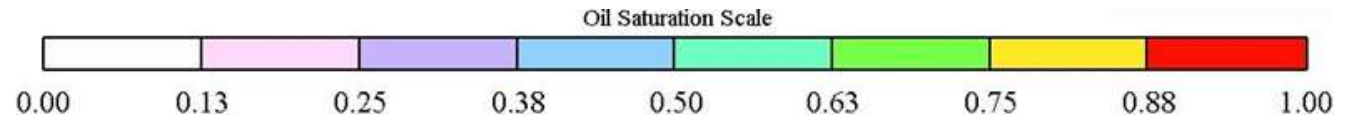

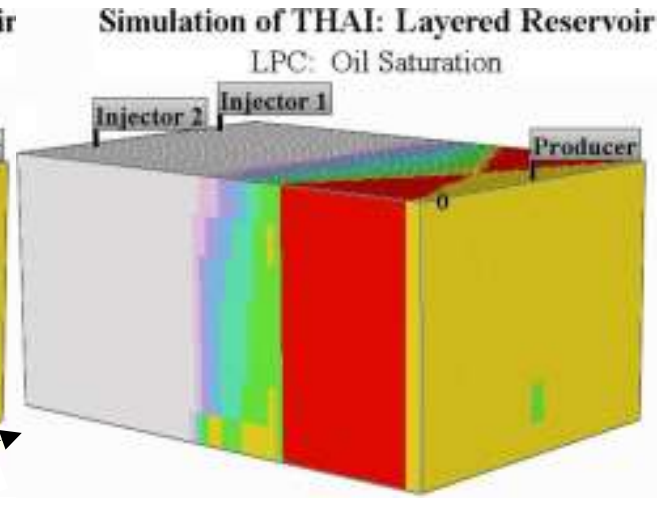

(b)

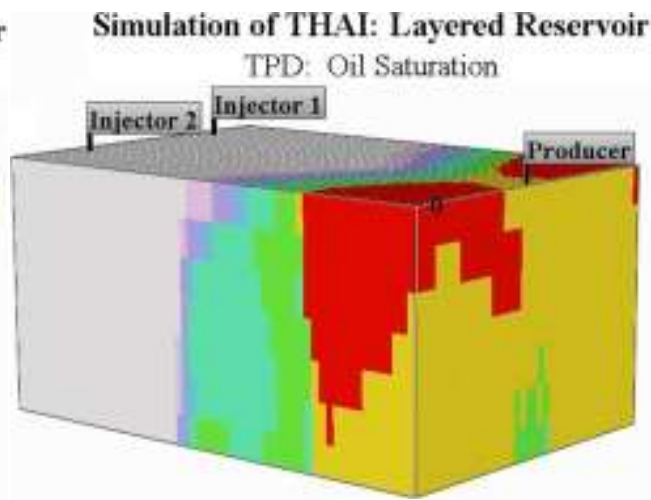

(d)

In simulations of THAI on the TPI model, two independent combustion fronts, one around each vertical injector, were initially developed until around 60 minutes from the start of the combustion. These fronts propagated rapidly downwards while tilting towards the toe of the HP. This was because the permeability increased vertical downwards, and, therefore, it was more difficult for the fronts to propagate horizontally. Around the toe of the HP, the fronts combined and advanced in the axial direction forming a wedge like protrusion along the HP. From Figure 13(c), it can be seen that at the base of the reservoir, and for two horizontal layers above that, the combustion front spread laterally at higher rate to sweep a large area. This shows that deploying THAI process into the TPI-type reservoir would result in a partially stable operation due to the rapid advancement of the combustion front inside the HP. At the end of six hours of combustion, the front inside the producer well had swept $46 \%$ of the 
total length of the HP well. It, therefore, follows that the combination of this finding, and the fact that the TPI model exhibits the lowest fuel availability among the four models, should result in poor oxygen consumption, and indeed, it was the lowest among the four layered models. However, since the highest permeability is at the bottom of the reservoir, the mobilised oil in the TPI reservoir easily flows into the HP. It should be noted that THAI is a gravity stabilised process implying that the mobilised oil must be gravity drained to the base of the reservoir first before flowing into the HP. As a result, for the same cumulative air injected, more oil was recovered from a TPI-type reservoir than for the LPC-type reservoir (see Table 2). Further, in contrast to the HPC model, but just as for the homogeneous experimental-scale model presented previously (Rabiu Ado et al., 2017), the TPI model had a rich oil bank ahead of the MOZ (see Figure 14(c)). In addition, the THAI simulations on the TPI model predicted the highest percentage recoverable oil at the end of the six hours of combustion.

The shape of the combustion front during simulation of THAI on the TPD reservoir model shown in Figure 13(d) is quite similar to that found for the HPC reservoir in Figure 13(a), except that the former is laterally broader and only covers a length of $34 \mathrm{~cm}$ axially. The combustion zone was conical in shape, with the narrow end pointing towards the heel of the HP. A large horizontal areal sweep was achieved around the vertical injectors implying that the highest permeability was located at the top horizontal plane. Compared with the findings for the TPI model in Figure 13(c), the combustion front in the TPD model did not reach the base of the reservoir, showing the impact of the lowest permeability being in the bottom horizontal plane. At the narrow end of the combustion front it travels at, essentially, the same rate, which is an indicator of stability. Therefore, the THAI process can operate in a stable manner in this type of reservoir. The predicted oxygen utilisation by THAI for the TPD model was 92.4\%, which was higher than that predicted for the TPI model by $0.8 \%$ (see Table 2). However, 0.7 \%OOIP less was recovered from the TPD reservoir model than from the TPI model. This could be explained by the restriction to mobilised oil flow into the HP caused by the presence of low permeability around the producer well. Amongst the four models, the TPD model had the highest fuel availability, which arose from the increased residence time of the mobilised oil. Figure 14(d) shows that, ahead of 
the MOZ, a rich oil bank (saturation of 88 to $100 \%$ ) was formed during THAI operation in the TPDtype reservoir. Generally speaking, the displaced oil zones for the TPI model (as seen in Figure 14(c)) and the TPD model (as seen in Figure 14(d)) were quite similar in form, except that the rich oil bank was formed in the corresponding particular regions where the permeability was the highest. Comparing the four models, it can be observed that the THAI process within the HPC model (Figure 14(a)) led to no such rich oil bank because it had a high permeability zone directly above the HP.

\subsection{Limits to safe operation of THAI with permeable cap-rock}

The operation of THAI, within the various reservoir models listed in Table 1, was simulated to determine what level of permeability of the cap-rock could be safely tolerated. As seen in Figure 15, at the end of the PIHC, $6.7 \%$ OOIP was recovered for the base case model, $\mathrm{C} 1$, with no cap-rock in the model cell (and adiabatic boundary conditions). This value is higher than the recovery achieved, over the same period of time, for both models $\mathrm{C} 2$ and $\mathrm{C} 3$ by around $10 \%$. For model C4, in which the caprock permeability $\left(K_{h} \& K_{v}\right)$ and porosity were one order of magnitude larger than in C3 (see Table 1), only $1.1 \%$ OOIP was recovered prior to air injection (as seen in Figure 15). When the vertical permeability $\left(\mathrm{K}_{\mathrm{v}}\right)$ was either 0.1 or $0.3 \mathrm{md}$, and the porosity was $1 \%$, the cap-rock was very tight such that only a negligible quantity of steam (less than 2 mol\%) could permeate through the rock. This can be seen in Figures 16(a) \& (b) which show, for models C2 and C3, respectively, that the injected steam was restricted to only the oil zone over the 104 days of the PIHC period. As a result, the heat transferred to the overlaying cap-rock during the steaming was mainly due to conduction. That, in turn, led to an increase in temperature from 29 to $115{ }^{\circ} \mathrm{C}$, above the initial reservoir temperature of $20{ }^{\circ} \mathrm{C}$, in both model C2 (Figure 16(d)) and C3 (Figure 16(e)), respectively. These are the reasons why the oil recovery, in models $\mathrm{C} 2$ and $\mathrm{C} 3$, is slightly lower than in the model containing (in effect) an impermeable, nonconductive cap-rock (i.e. C1). However, when the cap-rock $\mathrm{K}_{\mathrm{v}}$ and porosity were increased by a factor 10 , as in model $\mathrm{C} 4$, a significant quantity of steam escaped into the cap-rock, displacing all the preexisting gas above the toe of the vertical injector (see Figure 16(c)). This explains the reason for the 
very low oil recovery in this case in comparison with the base case model $\mathrm{C} 1$. Unlike in models $\mathrm{C} 2$ and $\mathrm{C} 3$, for model $\mathrm{C} 4$ the heat was transferred to the cap-rock via both conduction and convection, with the latter predominating. This suggestion is supported by the rise in the temperature of the steam swept zone at the end of the PIHC (see Figure 16(f)).

Figure 15. Oil recovery for simulations of THAI on reservoir models with cap-rocks of variable permeability. Details of the models C1-C4 are given in Table 1.

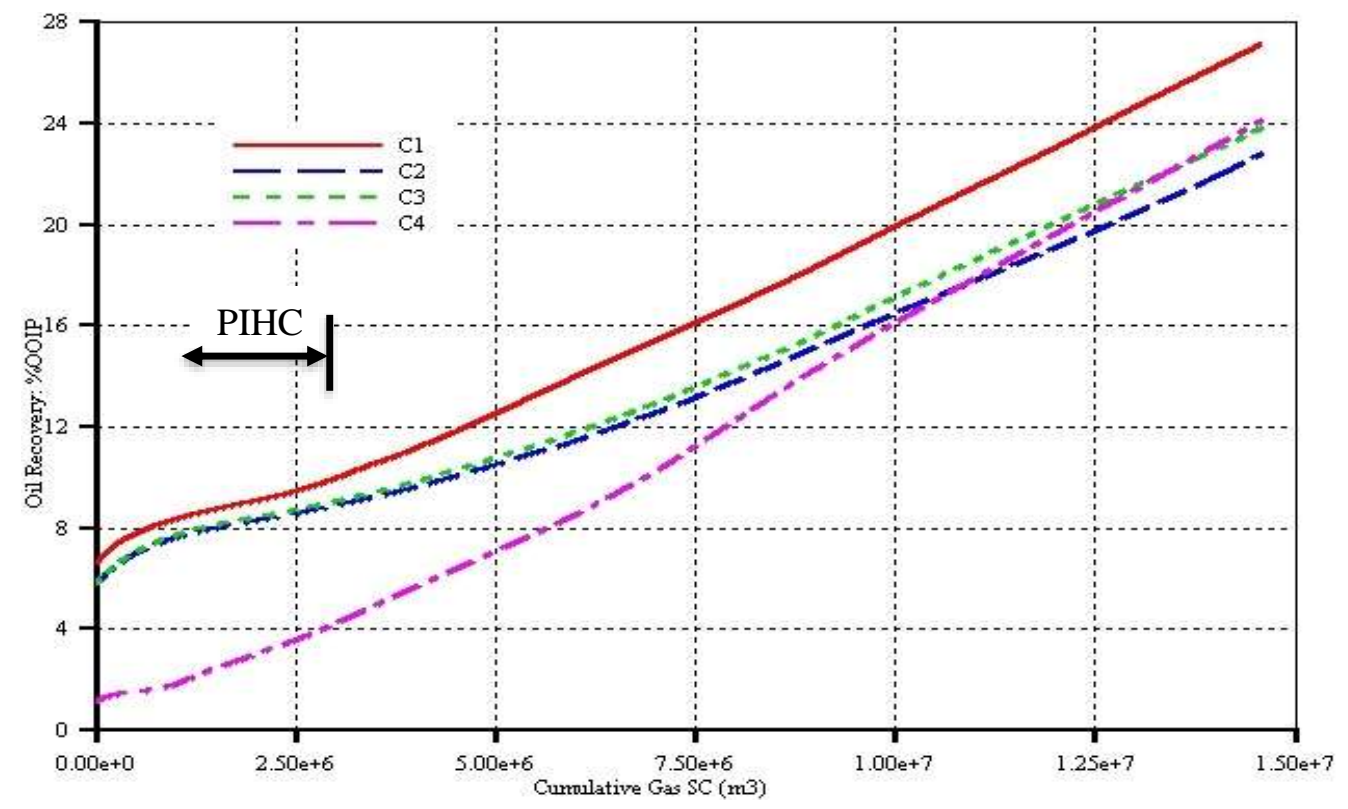

As can be seen from Figure 17, the inclusion of a permeable cap-rock in the reservoir models also resulted in a lower oil recovery throughout the additional two years of combustion. At the end of the simulated period, $27.2 \%$ OOIP had been recovered from model $\mathrm{C} 1$, which was higher than that recovered from model C2 by approximately $20 \%$. Also, the recovery from model C3 was $4.5 \%$ higher than that obtained from model $\mathrm{C} 2$. When the cap-rock permeability and porosity were increased by a factor of 10, the recovery in C4 was $1.3 \%$ higher than in C3. Similar observations can be made from the oil recovery curves, given in Figure 17, in which the production from model $\mathrm{C} 1$ is greater than that for models $\mathrm{C} 2$ and $\mathrm{C} 3$ throughout the combustion period. This is probably due to some of the heat generated from the combustion being conducted (i.e. in the case of $\mathrm{C} 2$ and $\mathrm{C} 3$ ) away to the cap-rock. In contrast, the produced oil curve for model $\mathrm{C} 4$ is steeper than those of the other three models from 
the end of the PIHC to the end of the combustion period. This was because less oil was mobilised earlier, during the PIHC, since then some steam escaped into the cap-rock, and also due to the decreased heat capacity of the cap-rock as the porosity is increased. All of the models demonstrated the same water production rate (not shown) during THAI operation, showing the presence of a permeable cap-rock (with the range of $\mathrm{K}_{\mathrm{v}}$ values considered) had no effect on the water production.

Figure 16. Steam mole fraction profiles along the vertical mid-plane for models (a) C2, (b) C3, and (c) $\mathrm{C} 4$, and temperature profiles along the vertical mid-plane for models (d) C2, (e) C3, and (f) C4, all at the end of the PIHC during simulations of THAI. The arrows represent oil flow vectors.

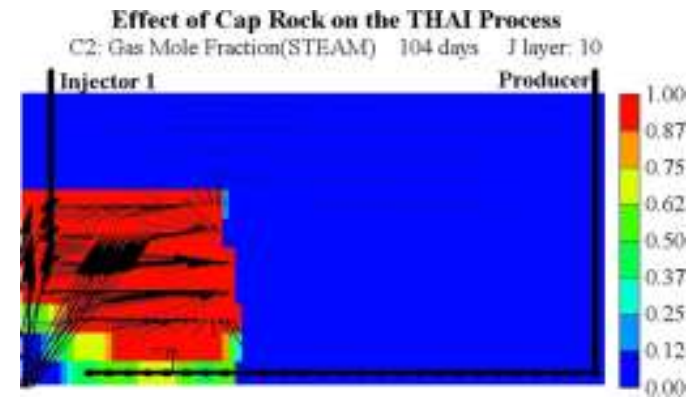

(a)

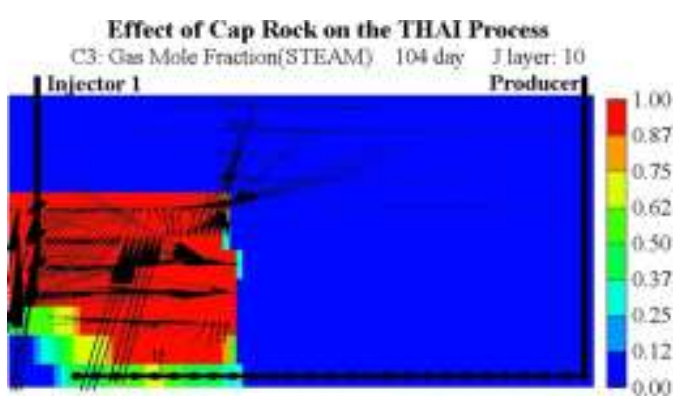

(b)

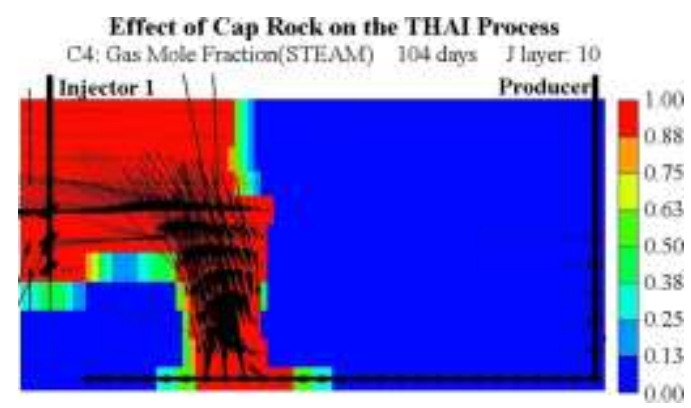

(c)

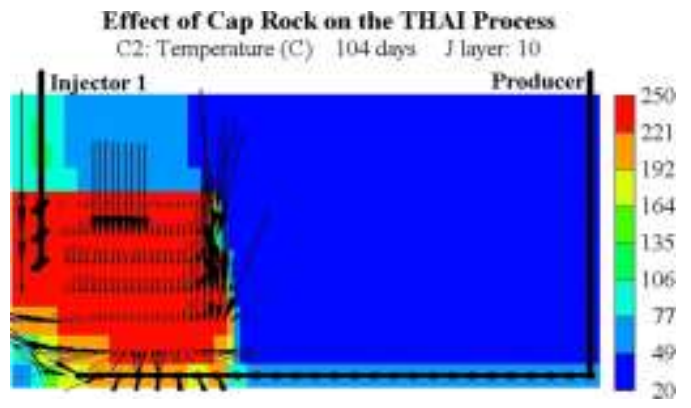

(d)

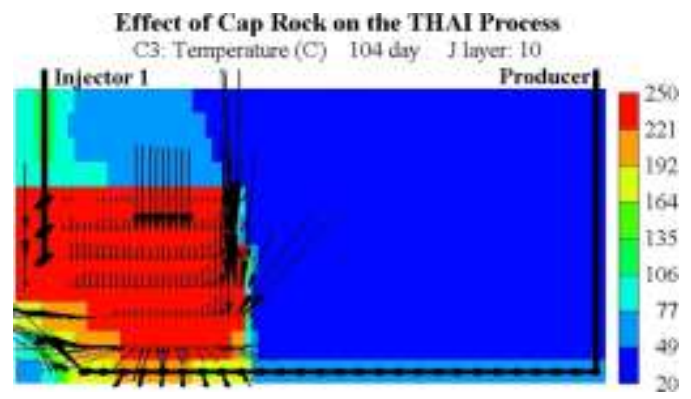

(e)

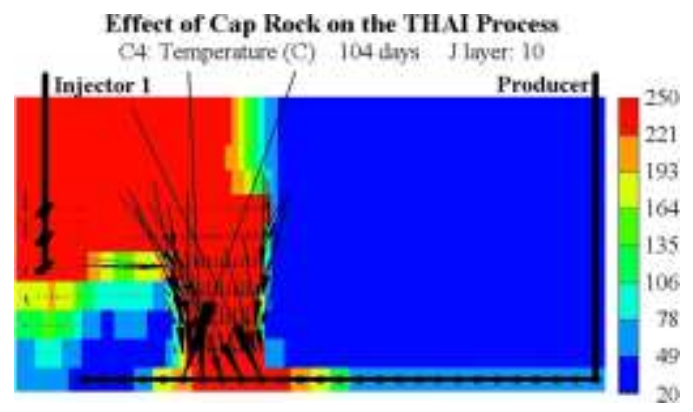

(f) 
Figure 17. Oil production rate for simulations of THAI on reservoir models (C1-4) with cap-rocks of differing permeability as shown in Table 1

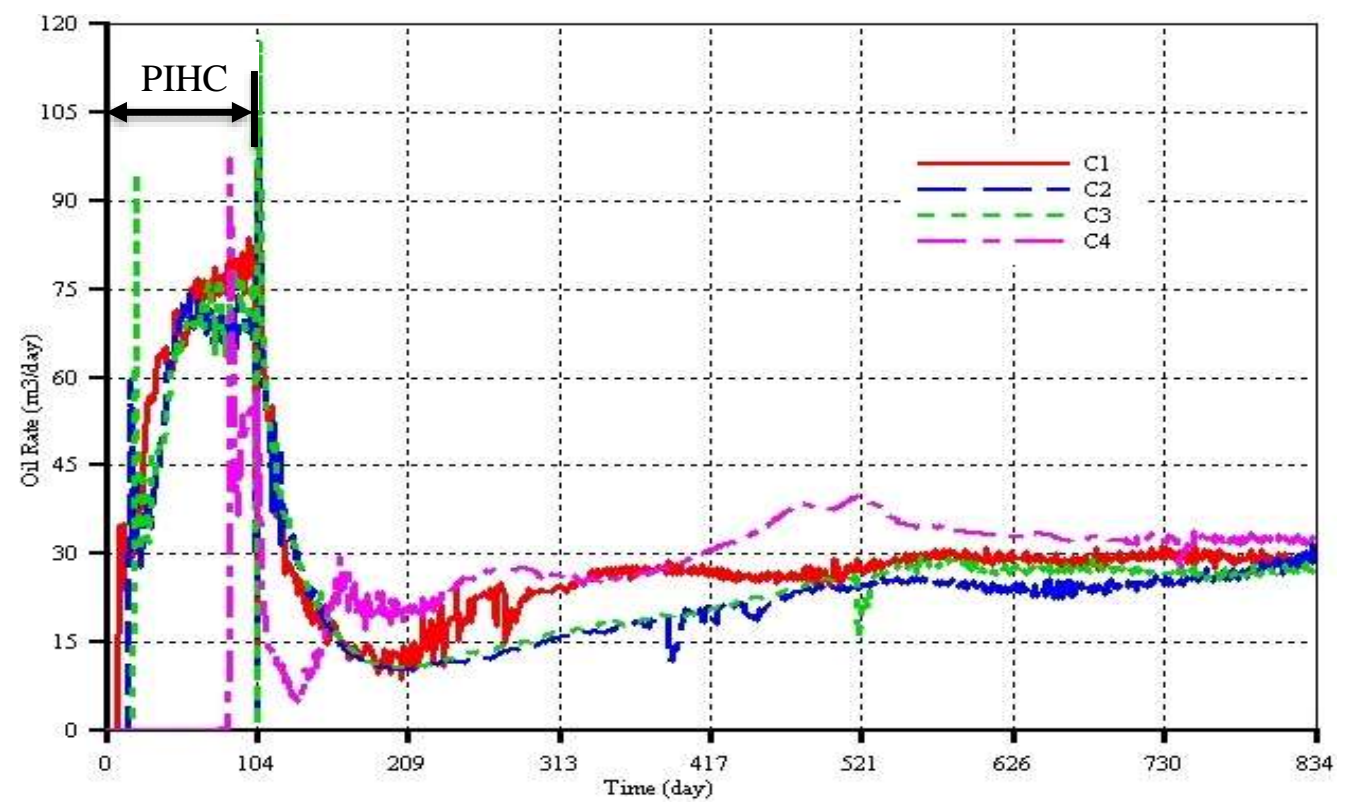

Figure 18 shows the degree of upgrading, in terms of API gravity, obtained during simulated THAI operation on the reservoir models with cap-rock. There was generally an increasing, but discontinuous, trend in API with increasing process time. This was because, as the combustion becomes more vigorous, the cracking rate increased, and thus more of the lighter pseudo-component was produced. The findings in Figure 18 also show that the higher the cap-rock vertical permeability, the longer it took before a marked steep increase in API was observed, as highlighted by the progressively later times for points $\mathrm{A}, \mathrm{B}$, and $\mathrm{C}$ indicating this transition. This can be attributed to the ability of the cap-rock in aiding heat distribution within the reservoir. Unlike in the case of models $\mathrm{C} 1, \mathrm{C} 2$, and $\mathrm{C} 3$, the large absolute vertical permeability and hence porosity of the cap-rock in model C4 resulted in a decrease in heat conduction and entrapping of the combustion gases within the oil reservoir. This, in turn, resulted in less thermal cracking of the mobilized oil. As a consequence, it took longer before an appreciable amount of upgrading to take place. 
Figure 18. API gravity for produced oil in simulations of THAI on reservoir models (C1-4) with caprocks of differing permeability as shown in Table 1

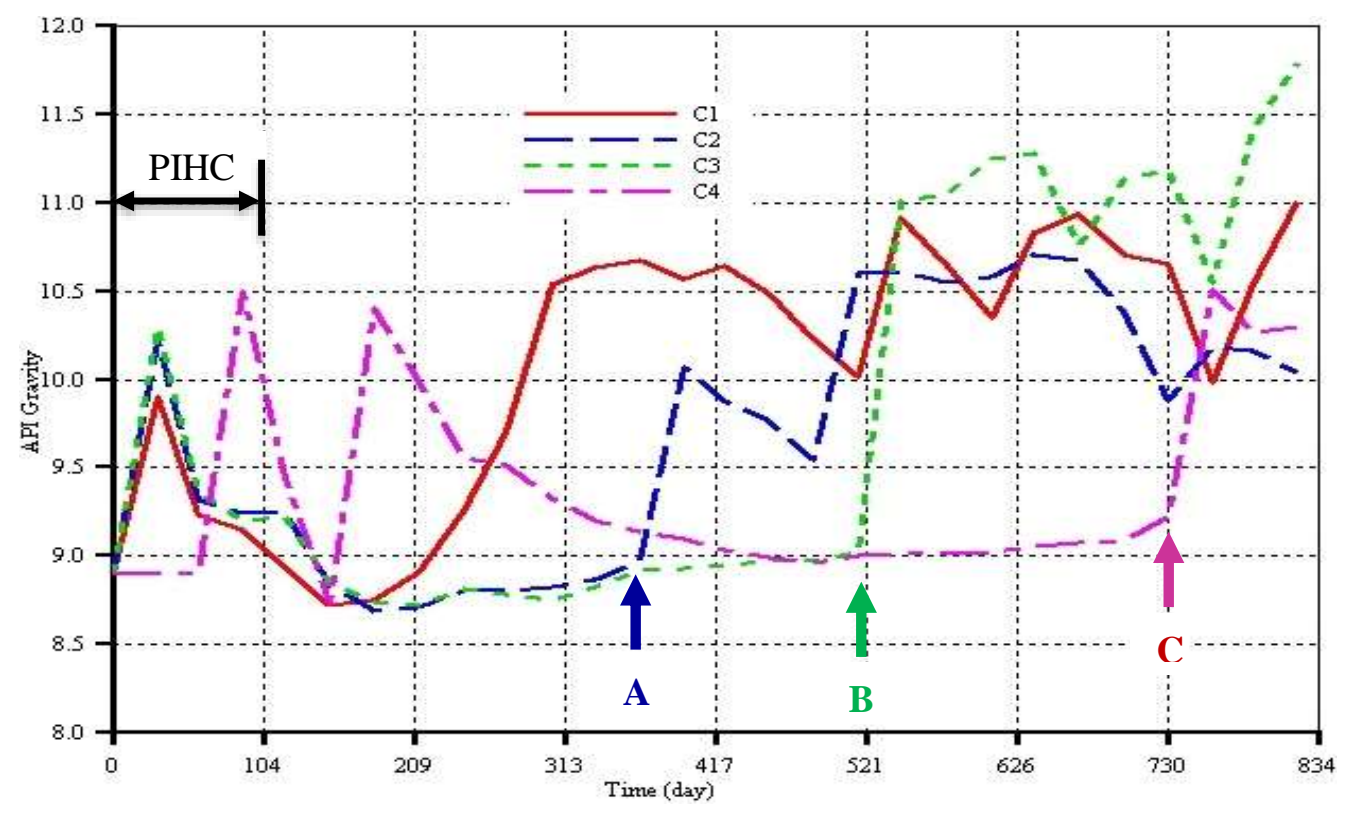

The data given in Table 3 shows that oxygen production increased with an increase in the $\mathrm{K}_{\mathrm{v}}$ value of the cap-rock. However, even for model $\mathrm{C} 4$, that with the highest cap-rock vertical permeability, the injected oxygen produced was only $1.33 \mathrm{~mol} \%$ of that injected. It is noted that the cumulative oxygen production rate (not shown) suggested some very minor process variability, since the oxygen production took place intermittently. However, the very low absolute values for oxygen production indicate that, overall, the THAI process operated stably even where the cap-rock was permeable, since the produced oxygen did not reach the breakthrough level. 
Table 3. Oxygen utilisation as mole percent of the cumulative oxygen injected for simulations of THAI on reservoir models C1-4 with variable cap-rock properties given in Table 1.

\begin{tabular}{|c|c|c|c|}
\hline Model & Accumulated (mol\%) & Produced (mol\%) & Utilised (mol\%) \\
\hline C1 & 0.96 & 0.38 & 98.66 \\
\hline C2 & 0.95 & 0.42 & 98.63 \\
\hline C3 & 0.78 & 0.68 & 98.54 \\
\hline C4 & 0.98 & 1.33 & 97.70 \\
\hline
\end{tabular}

The oxygen profiles obtained for along the vertical mid-plane of the reservoir models (shown in Figures 19(a)-(c)), and the adjacent vertical plane (shown in Figures 19(d)-(f)), illustrate the shape of the combustion front during THAI operation. Behind the front, a small amount of oxygen accumulates before it is consumed on reaching the combustion zone. However, as remarked above, a small amount of oxygen still bypassed the combustion front through the low-fuel-concentration zones to reach the HP well. In addition to that, some of this oxygen escaped into the cap-rock, possibly via the low temperature region or via zones that contain no coke (see Figure 19). Due to the low vertical permeability in model C2 (Figure 19(a)\&(b)), most of the escaped oxygen (despite being very small in amount) became entrapped within the cap-rock. That is why there was an increase in oxygen production by $10 \%$ over that produced from model $\mathrm{C} 1$. When the $\mathrm{K}_{\mathrm{v}}$ value of the cap-rock in $\mathrm{C} 2$ was tripled (i.e. to form $\mathrm{C} 3$ ), a larger amount of oxygen escaped into the cap-rock. However, most of it was ultimately also produced, thereby implying an increase in oxygen production by $80 \%$ of that produced in $\mathrm{C} 1$ (as seen in Table 3 ). An additional increase in both $\mathrm{K}_{\mathrm{v}}$ and $\mathrm{K}_{\mathrm{h}}$, to create model $\mathrm{C} 4$, resulted in an increase in the accumulated and produced oxygen concentration since then even higher amounts escaped into the cap-rock. Further, if the cap-rock is permeable combustion gases may also escape. If a gas reservoir were to lie above the heavy oil and the heavy oil cap-rock leaked combustion gases this could be an issue. 
Figure 19. Oxygen mole fraction profiles along the vertical mid-plane of models (a) $\mathrm{C} 2$, (b) $\mathrm{C} 3$, and (c) C4, and along the vertical adjacent planes of models (d) C2, (e) C3, and (f) C4.

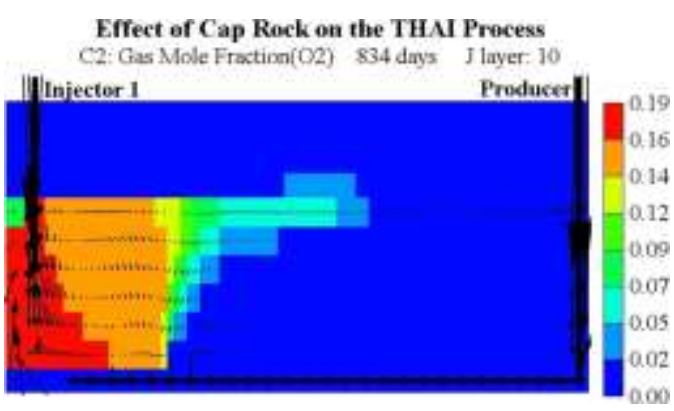

(a)

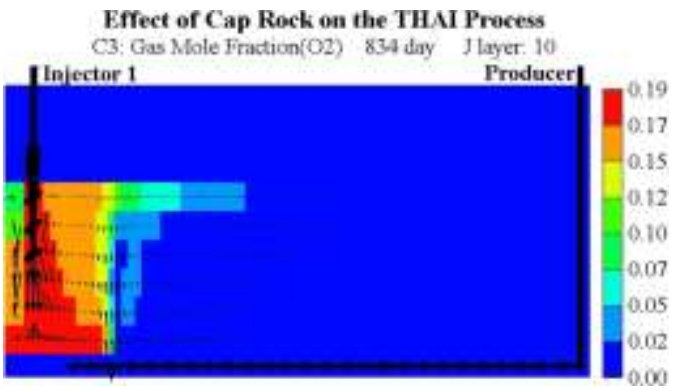

(b)

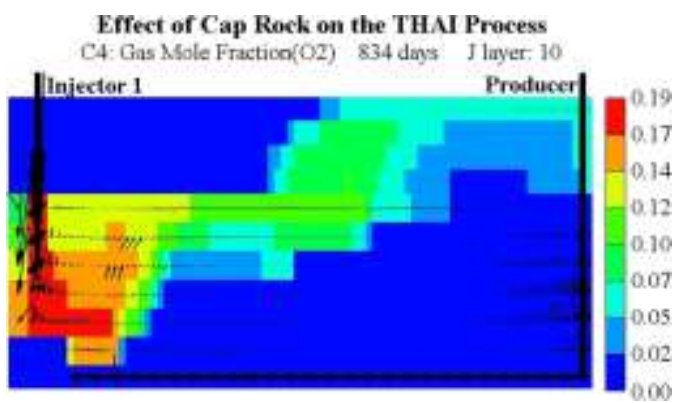

(c)

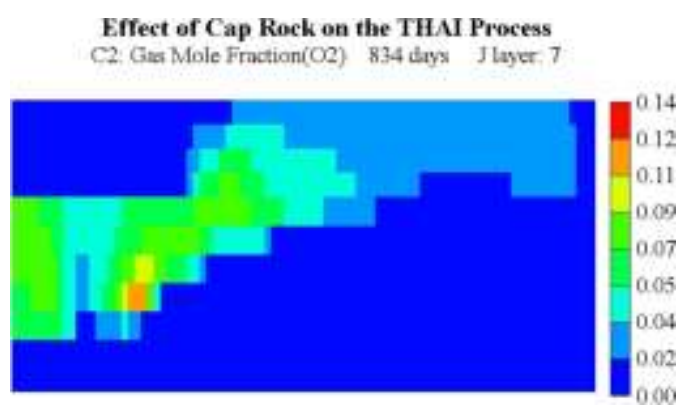

(d)

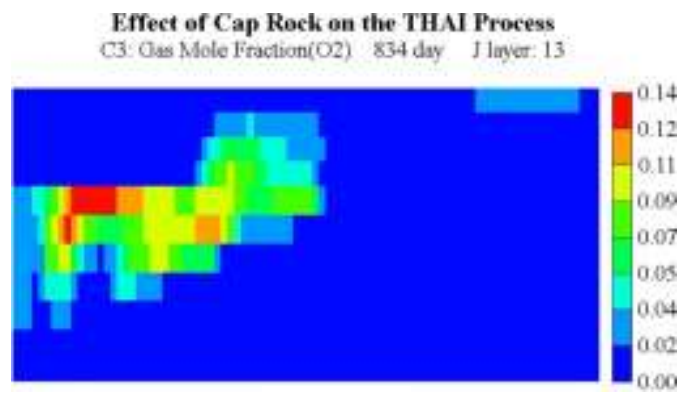

(d)

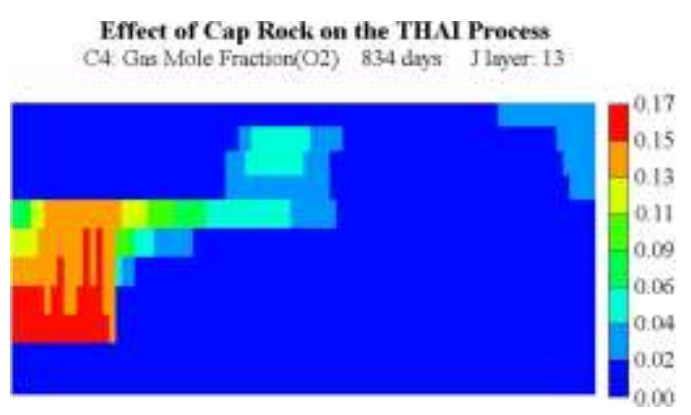

(f)

The oil drainage pattern during operation of the THAI process was, broadly, not affected by the variability in cap-rock present, as evident from Figure 20, but viscosity is profoundly affected. The quantity of oil mobilised and produced from the three models was only marginally different (i.e. varying by a maximum of 5.5\%). However, the viscosity of the oil within the cold oil zone (i.e. the oil zone downstream of the combustion zone) for models with cap-rocks showed a dramatic decrease in viscosity compared to when no cap-rock was included in the reservoir model. The impact of this difference can 
be seen in Figures 20(a)-(d), since the oil saturation becomes richer (i.e. 88 to 100\%) compared to the initial saturation of $80 \%$. From the temperature distribution, it was found the decrease in viscosity was caused by an increase in heat distribution. This occurred because the heat conducted (i.e. in the case of models $\mathrm{C} 2$ and $\mathrm{C} 3$ ) to the cap-rock from around the combustion zone was rapidly transported downstream, towards the heel end of the HP well. This was the reason why the oil saturation in the top horizontal oil layer, just below the oil-cap-rock interface, was substantially reduced to $\leq 50 \%$ (see Figures 20(a)-(d)). This finding implies that there was a top-down drainage of oil from zones not directly affected by the "bulldozing effect" of the combustion front, as indicated by the oil flux vectors superimposed on the oil saturation profiles in Figure 20. Comparing the temperature distribution within models $\mathrm{C} 1, \mathrm{C} 2$, and $\mathrm{C} 3$, it was found that the minimum temperatures of the cold oil layer, at 834 days, were approximately 20,42 , and $45{ }^{\circ} \mathrm{C}$ for models $\mathrm{C} 1, \mathrm{C} 2$, and $\mathrm{C} 3$ respectively. This shows that the tripling of the $\mathrm{K}_{\mathrm{v}}$ value (i.e. from that in $\mathrm{C} 2$ to that in $\mathrm{C} 3$ ) enhances the heat transfer in the cold oil zone, most probably by convection. From these observations, it can be inferred that the inclusion of the caprock in reservoir models for the simulation of THAI is essential to accurately mimic the exact heat distribution into the oil zone of the reservoir.

Since both the permeability $\left(\mathrm{K}_{\mathrm{v}}\right.$ and $\left.\mathrm{K}_{\mathrm{h}}\right)$ and the porosity of the cap-rock were increased by a factor of 10 in model $\mathrm{C} 4$, the effectiveness of the cap-rock in aiding heat distribution into the oil reservoir was reduced. This was because convection then became the dominant mechanism through which heat was distributed due to the larger pore volume easily accessible by the combustion gases. Additionally, the contact surface between the rock particles was reduced with the increase in porosity thereby reducing the effectiveness of heat transfer via conduction. That is why there was then no top-down oil drainage from the top horizontal oil layer below the cold oil zone as depicted by the absence of oil flux vectors in Figures 20(e) and (f). This can be noted by observing that the oil saturation there has a value of $\geq 75$ $\%$. Further, on comparing the oil saturation profiles for the three models $\mathrm{C} 1, \mathrm{C} 2$, and $\mathrm{C} 3$, it can be seen that model C3 has the smallest rich oil bank area, which is due to absence of top-down oil drainage in 
the cold oil zone. The minimum temperature in the cold oil zone in $\mathrm{C} 4$ was found to be roughly $24{ }^{\circ} \mathrm{C}$, which is still higher than in the absence of cap-rock.

Figure 20. Oil saturation profiles along the vertical mid-plane, and adjacent vertical mid-planes, for reservoir models $\mathrm{C} 2(\mathrm{a} \& \mathrm{~b}), \mathrm{C} 3(\mathrm{c} \& \mathrm{~d}) \mathrm{C} 3$, and $\mathrm{C} 4(\mathrm{e} \& \mathrm{f})$, respectively, during simulations of THAI operation in reservoirs with different cap-rocks shown in Table 1.

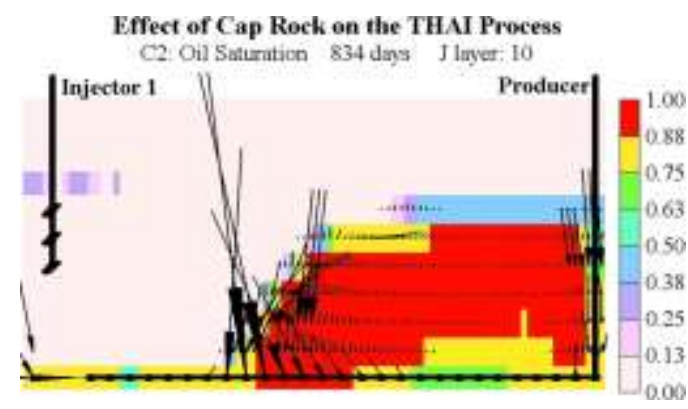

(a)

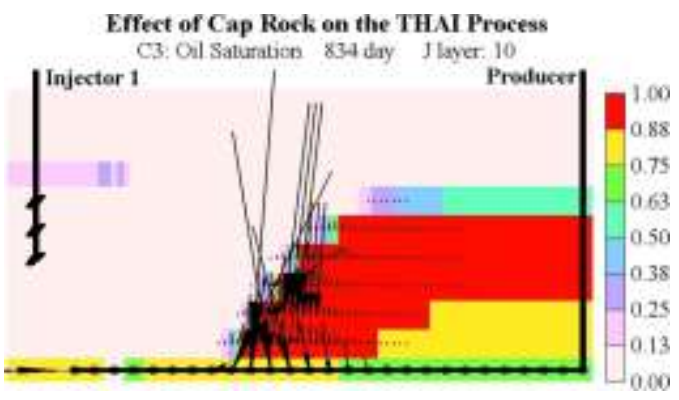

(c)

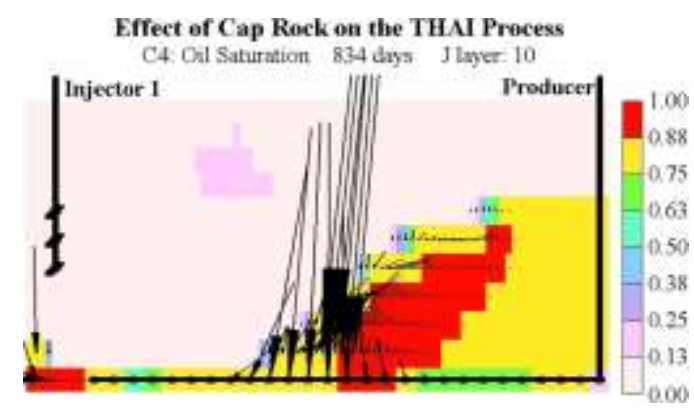

(e)

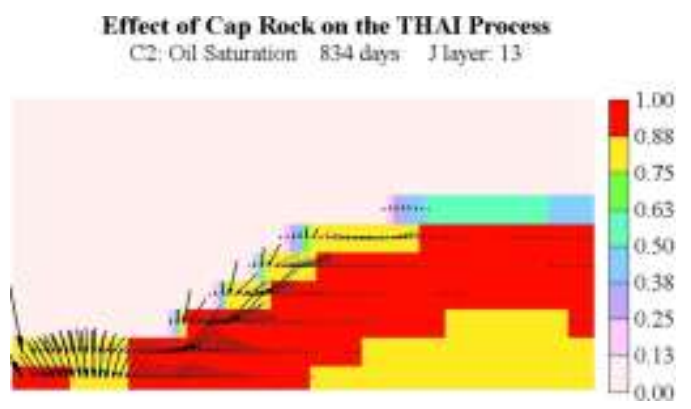

(b)

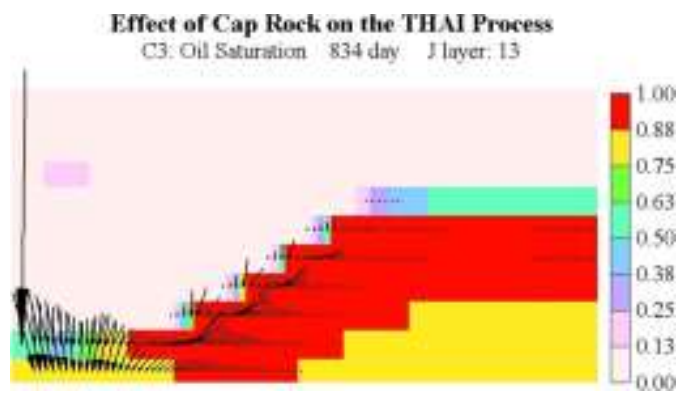

(d)

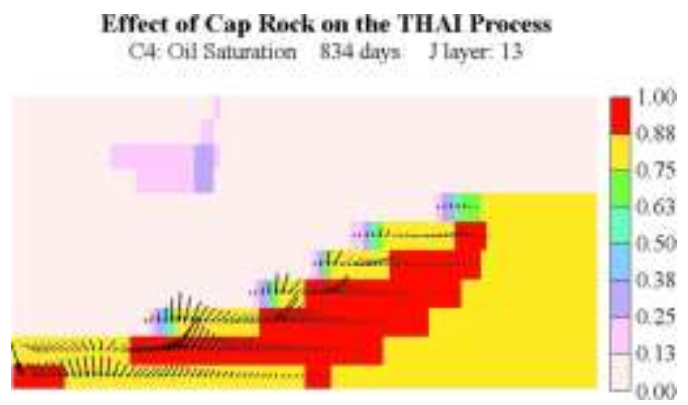

(f)

\section{CONCLUSIONS}

It has been shown that the THAI process can still operate stably for a wide range of reservoir permeabilities, and types and degrees of geological heterogeneity. However, the degree of heterogeneity 
affects the pattern of oxygen utilisation. Geological heterogeneity affects fuel concentration, and thus the progression of the combustion front, making the process potentially harder to control, with the possibility of oxygen breakthrough. For example, the presence of a significant fraction of low permeability zones increases the risk of oxygen breakthrough via channelling into the HP. However, the presence of shale layers did not to lead to oxygen breakthrough due to preferential channelling to the HP. Reservoirs with shale layers had higher fractional oil recovery, but obtained with larger air-tooil ratio. In general, it is necessary to modify air-flow rate depending on the nature of the heterogeneity eg if seen in seismic surveys. The type of heterogeneity also determines whether an oil bank forms ahead of the mobile oil zone.

The presence of a random distribution of low permeability zones led to a breakdown of the symmetry of the combustion front propagation, even in the case of two VI wells. This suggests simplifying assumptions based on symmetry are not applicable even in systems with that apparent symmetry but in the presence of heterogeneities.

It has been seen that careful well placement, respecting the particular geological heterogeneity in the reservoir, is essential to ensure THAI operates efficiently. In layered reservoirs where the high permeability zone is in the middle of the reservoir (HPC model), implementing the THAI process with the wells arranged in an SLD arrangement, and the HP located in the highest permeability zone, would result in unstable operation. However, implementing the THAI process to recover oil from a layered reservoir with a low permeability zone in the mid-plane ( LPC model) would provide safe operation, since oxygen production is delayed. It was found that the main negative impact that low permeability along the central axis has on the THAI process is the lowering of the quantity of mobilised oil reaching the producer well. 
It has been shown that the proper representation of the cap-rock in reservoir models for the field-scale simulation of THAI is essential in order to accurately mimic the full pattern of heat distribution into the oil zone of the reservoir, and, thence, fuel lay-down. However, the THAI process will remain stable even with a significant permeability in the reservoir cap-rock. However, vertical permeabilities above $\sim 1-3 \mathrm{mD}$ will lead to the significant loss of combustion gases from the reservoir.

\section{ACKNOWLEDGEMENTS}

Thanks are due to the Computer Modelling Group (CMG) for supplying comprehensive STARS simulation software. 


\section{REFERENCES}

Ado, M. R., Greaves, M., Rigby, S.P. (2018). Effect of pre-ignition heating cycle method, air injection flux, and reservoir viscosity on the THAI heavy oil recovery process. Journal of Petroleum Science and Engineering. 166: 94-103.

Akkutlu, I.Y and Yortsos, Y.C. ( 2005). The effect of heterogeneity on in-situ combustion: Propagation of combustion fronts in layered porous media. SPE Journal, 10: 394-404.

Barr, D. (2007). Conductive faults and sealing fractures in the West Sole gas fields, southern North Sea, in:Jolley, S.J., Barr, D., Walsh, J.J., and Knipe, R.J. (Eds.), Structurally Complex Reservoirs, London, Geological Society, pp.431-452.

Chen, Q. (2009). Assessing and improving steam-assisted gravity drainage: Reservoir heterogeneities, hydraulic fractures, and mobility control foams. $\mathrm{PhD}$ thesis, Stanford University. https://pangea.stanford.edu/ERE/pdf/pereports/PhD/ChenQing09.pdf

CMG (2012). Advanced Process and Thermal Reservoir Simulator, Calgary, Alberta Canada, Computer Modelling Group Ltd.

Deschamps, R., Guy, N., Preux, C. and Lerat, O. (2012). Analysis of Heavy Oil Recovery by Thermal EOR in a Meander Belt: From Geological to Reservoir Modeling. Oil Gas Sci. Technol. - Rev. IFP Energies nouvelles, 67, 999-1018. 
Fatemi, S.M. (2012). The effect of geometrical properties of reservoir shale barriers on the performance of steam-assisted gravity drainage (SAGD). Energy Sources Part A 34:2178-2191.

Le Ravalec, M., Morlot, C., Marmier, R., and Foulon, D. (2009). Heterogeneity impact on SAGD performance in mobile heavy oil reservoirs. Oil \& Gas Sci. Tech. 64: 469-476.

Li, H., Vink, J. C. and Alpak, F. O. (2015). An Efficient Multiscale Method for the Simulation of Insitu Conversion Processes. SPE Journal 20: 579-593.

Li, H., Vink, J. C. and Alpak, F. O. (2016). A Dual-Grid Method for the Upscaling of Solid-Based Thermal Reactive Flow, With Application to the In-Situ Conversion Process. SPE Journal 21: 20972111.

Mossop, G. D. 1980. Geology of the Athabasca oil sands. Science, 207, 145-152.

Nguyyen, T.B.N., Dang, T.Q.C., and Chen, Z. (2014). Effects of reservoir heterogeneities, thief zone, and fracture systems on the Fast-SAGD process. Energy Sources Part A 36:1710-1725.

Petrobank, 2008. Whitesands Experimental Project IETP Annual Report http://www.energy.alberta.ca/3040.asp [accessed 20/10/17]. 
Petrobank, 2009. Whitesands Experimental Project IETP Annual Report http://www.energy.alberta.ca/3261.asp [accessed 20/10/17].

Pooladi-Darvish, M. and Mattar, L. ( 2002). SAGD operations in the presence of overlying gas cap and water layer-effect of shale layers. Journal of Canadian Petroleum Technology, 41.

Rabiu Ado, M. (2017). Numerical simulation of heavy oil and bitumen recovery and upgrading techniques. PhD thesis, University of Nottingham. http://eprints.nottingham.ac.uk/41502/

Rabiu Ado, M., Greaves, M., Rigby, S.P. (2017a). Dynamic Simulation of the Toe-to-Heel Air Injection Heavy Oil Recovery Process. Energy \& Fuels 3:1276-1284.

Su, Y., Wang, J., and Gates, I.D. (2013). SAGD well orientation in point bar sand deposit affects performance. Eng. Geol. 157: 79-92.

Su, Y., Wang, J., and Gates, I.D. (2014). Orientation of a pad of SAGD well pairs in an Athabasca point bar deposit affects performance. Mar. \& Pet. Geol. 54: 37-46. 